\title{
First-principles study of thin magnetic transition-metal silicide films on $\mathrm{Si}(001)$
}

\author{
Hua Wu, Peter Kratzer, and Matthias Scheffler \\ Fritz-Haber-Institut der Max-Planck-Gesellschaft, Faradayweg 4-6, D-14195 Berlin, Germany \\ (Received 20 April 2005; revised manuscript received 2 August 2005; published 27 October 2005)
}

\begin{abstract}
In order to combine silicon technology with the functionality of magnetic systems, a number of ferromagnetic (FM) materials have been suggested for the fabrication of metal/semiconductor heterojunctions. In this work, we present a systematic study of several candidate materials in contact with the Si surface. We employ density-functional theory calculations to address the thermodynamic stability and magnetism of both pseudomorphic CsCl-like $M$ Si $(M=\mathrm{Mn}, \mathrm{Fe}, \mathrm{Co}, \mathrm{Ni})$ thin films and Heusler alloy $M_{2} \mathrm{MnSi}(M=\mathrm{Fe}, \mathrm{Co}, \mathrm{Ni})$ films on $\mathrm{Si}(001)$. Our calculations show that $\mathrm{Si}$ termination of the $M$ Si films is energetically preferable during epitaxy since it minimizes the energetic cost of broken bonds at the surface. Moreover, we can explain the calculated trends in thermodynamic stability of the $M$ Si thin films in terms of the $M$-Si bond strength and the $M 3 d$ orbital occupation. From our calculations, we predict that ultrathin MnSi films are FM with sizable spin magnetic moments at the Mn atoms, while FeSi and NiSi films are nonmagnetic. However, CoSi films display itinerant ferromagnetism. For the $M_{2} \mathrm{MnSi}$ films with Heusler-type structure, the MnSi termination is found to have the highest thermodynamic stability. In the FM ground state, the calculated strength of the effective coupling between the magnetic moments of $\mathrm{Mn}$ atoms within the same layer approximately scales with the measured Curie temperatures of the bulk $\mathrm{M}_{2} \mathrm{MnSi}$ compounds. In particular, the $\mathrm{Co}_{2} \mathrm{MnSi} / \mathrm{Si}(001)$ thin film has a robust FM ground state as in the bulk, and is found to be stable against a phase separation into $\mathrm{CoSi} / \mathrm{Si}(001)$ and $\mathrm{MnSi} / \mathrm{Si}(001)$ films. Hence this material is of possible use in FM-Si heterojunctions and deserves further experimental investigations.
\end{abstract}

DOI: 10.1103/PhysRevB.72.144425

PACS number(s): 75.70.-i, 73.20.At, 68.35.Md

\section{INTRODUCTION}

Metal-semiconductor heterojunctions have received much attention in the context of magnetoelectronics or spintronics because they could open up the possibility to inject a spinpolarized current from a ferromagnetic (FM) metal into a semiconductor. This is a prerequisite for anticipated future electronic devices making use of spin-polarized carriers. ${ }^{1}$ In this paper, we present theoretical investigations of thin films for two materials classes relevant in this context, namely, transition metal (TM) monosilicides $M$ Si $(M=\mathrm{Mn}, \mathrm{Fe}, \mathrm{Co}$, $\mathrm{Ni}$ ) in the $\mathrm{CsCl}$ crystal structure and Heusler alloys $M_{2} \mathrm{MnSi}$ $(M=\mathrm{Fe}, \mathrm{Co}, \mathrm{Ni})$. The two materials classes are closely related in their crystal structure. Pictorially, one can think of $M_{2} \mathrm{MnSi}$ films as being formed by the substitution of $\mathrm{Mn}$ for half of the $\mathrm{Si}$ atoms in each $\mathrm{Si}$ layer of the CsCl-like $M \mathrm{Si}$ $(M=\mathrm{Fe}, \mathrm{Co}, \mathrm{Ni})$ films. Both materials classes are of potential interest for spintronics applications. Some Heusler alloys, such as $\mathrm{Co}_{2} \mathrm{MnZ}(Z=\mathrm{Si}, \mathrm{Ge}, \mathrm{Sn})$ are ferromagnets even well above room temperature, and are predicted by band theory to be magnetic half-metals, i.e., the Fermi energy lies in a region of partially occupied bands for one spin channel, while lying in a gap of the density of states in the other. ${ }^{2-4}$ Therefore half-metallic Heusler alloys can in principle provide $100 \%$ spin-polarized carriers, and could thus serve as spin filters in future spintronics devices. However, also the structurally simpler monosilicides have a potential to be applied in spintronics devices: Recently, we have shown that thin MnSi films on $\mathrm{Si}(001)$ possess sizable magnetic moments at the $\mathrm{Mn}$ atoms, ${ }^{5}$ despite the fact that bulk MnSi (in the corresponding hypothetical $\mathrm{CsCl}$ crystal structure) is nonmagnetic. Moreover, calculations of $\mathrm{CoSi}$ in $\mathrm{CsCl}$ crystal structure find this (metastable) compound to be ferromagnetic.
This motivated us to study systematically both the structural and magnetic properties of late TM monosilicides films. In addition, mixed TM silicides have also attracted interest, since evidence has been given that FeSi could be made ferromagnetic by doping with Co. 6,7

From the viewpoint of applications, it is highly desirable to grow well-defined FM metallic films on the most common semiconductor, silicon, in particular on the technologically relevant $\mathrm{Si}(001)$ surface. For this reason, we concentrate in the present paper on pseudomorphic thin films of monosilicides and Heusler alloys on $\mathrm{Si}(001)$. For epitaxial growth, the monosilicides in CsCl-like crystal structure are particularly attractive: We find that the $\mathrm{CsCl}$ structure is a metastable phase of the monosilicides, only moderately higher in energy than the ground-state crystal structure, and it is closely lattice matched with $\mathrm{Si}(001)$. Moreover, such $\mathrm{CoSi}$ and NiSi crystals have been found to be "supersoft" materials, ${ }^{8}$ i.e., there is a range of elastic deformations with very little energetic cost. The Heusler alloys show a somewhat larger lattice mismatch with $\mathrm{Si}(001)$ of about $4 \%$. Apart from good lattice match, flat and atomically sharp interfaces are of crucial importance for efficient spin injection. In this context, it is noteworthy that disilicide films have been grown with atomically sharp interfaces to $\mathrm{Si}(111)$ and $\mathrm{Si}(100)$. The $\mathrm{CaF}_{2}$ crystal structure of disilicides is similar to the $\mathrm{CsCl}$ crystal structure of monosilicides (it results if each second metal site in the $\mathrm{CsCl}$ structure is left vacant). This suggests that film growth with atomically sharp interface should also be possible for the monosilicides films. In practice, first a buffer layer of the disilicide is grown, followed by growth of the monosilicide film. With this strategy, $\mathrm{CsCl}-$ like FeSi and CoSi films have already been grown on $\mathrm{Si}(111)$ by von Känel et al. ${ }^{9,10}$ 
(a)

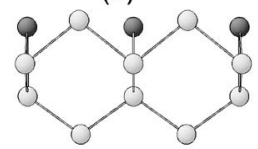

(b)

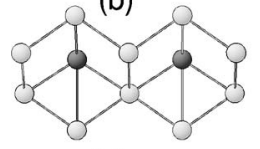

(c)

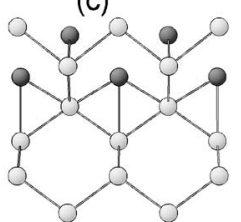

(d)

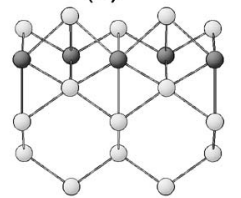

(e)

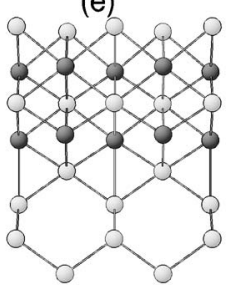

(f)

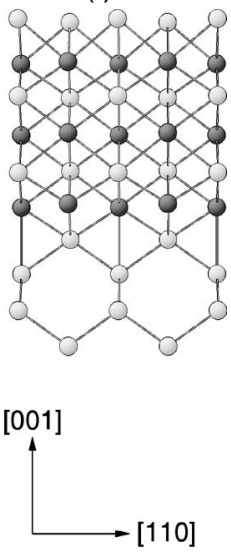

FIG. 1. Side view of various $M=\mathrm{Mn}, \mathrm{Fe}, \mathrm{Co}$, or $\mathrm{Ni}$ films on $\mathrm{Si}(001)$ (half of the slab), with $0.5 \mathrm{ML} M$ in (a) the first- or (b) second-layer interstitial sites, 1 ML $M$ (c) in a mixed layer or (d) in a Si- $M$ sandwich, or (e) 2 ML or (f) 3 ML $M$ CsCl-like sandwich structures. Black balls represent $M$ and gray balls $\mathrm{Si}$ atoms. The bonds shorter than $2.65 \AA$ are shown.

While theoretical investigations of CsCl-like $M \mathrm{Si}$ thin films on $\mathrm{Si}(001)$ are scarce, ${ }^{11}$ a group of studies addressing the initial reaction processes of TM adatoms with the $\mathrm{Si}$ substrate report that $\mathrm{Mn}, \mathrm{Co}$, and $\mathrm{Ni}$ adatoms prefer subsurface sites. ${ }^{5,12-14}$ Heusler alloy films have been studied experimentally mostly in view of their application in tunneling magnetoresistance devices. ${ }^{15-17}$ Concerning epitaxial growth on semiconductor substrates, results for thin $\mathrm{Co}_{2} \mathrm{MnGe}$ (Ref. 18) and $\mathrm{Co}_{2} \mathrm{MnSi}$ (Ref. 19) films on $\mathrm{GaAs}(001)$ have been reported. From the theoretical side, calculations of the $\mathrm{Co}_{2} \mathrm{MnSi}(001)$ surface, ${ }^{20,21}$ as well as of the interface between $\mathrm{Co}_{2} \mathrm{MnGe}$ and GaAs(001) (Refs. 22 and 23) have been performed.

In the present paper, we identify the trends in chemical bonding, thermodynamic stability, and magnetism of the $M \mathrm{Si}$ and $M_{2} \mathrm{MnSi}$ thin films. Most importantly, our calculations predict that, in addition to ultrathin $\mathrm{FM} \mathrm{MnSi} / \mathrm{Si}(001)$ films, ${ }^{5}$ the $\mathrm{CoSi} / \mathrm{Si}(001)$ thin films are also $\mathrm{FM}$; and that $\mathrm{Co}_{2} \mathrm{MnSi} / \mathrm{Si}(001)$ films have a robust FM ground state.

\section{COMPUTATIONAL DETAILS}

The present DFT calculations were performed using the all-electron full-potential augmented plane-wave plus localorbital method. ${ }^{24}$ The generalized gradient approximation ${ }^{25}$ (GGA) was adopted for the exchange-correlation potential, since it has been shown ${ }^{26,27}$ that GGA gives a better description for both transition metals and their silicides than the local-spin-density approximation. The $M \mathrm{Si}$ or $M_{2} \mathrm{MnSi}$ thin films on $\mathrm{Si}(001)$ were modeled by a slab consisting of eight successive $\mathrm{Si}(001)$ layers and the $M \mathrm{Si}$ (see Fig. 1) or $M_{2} \mathrm{MnSi}$ layers (see Sec. III C) on both sides, in order to retain the inversion symmetry. The GGA calculated equilibrium lattice constant $(5.48 \AA$ ) of bulk $\mathrm{Si}$ is used for the $\mathrm{Si}(001)$ substrate. A supercell with about $10-11 \AA$ vacuum between the slabs, and with a lateral $(1 \times 1)$ periodicity $^{5}$ (lat- tice constant of $3.87 \AA$ ) was used. Note that $\theta=1 \mathrm{ML}$ (monolayer) coverage of $M$ refers to two $M$ adatoms per $(1 \times 1)$ cell on either side of the slab. The muffin-tin radii are chosen to be $1.11 \AA$ for $\mathrm{Mn}$, as used in our previous calculations, ${ }^{5}$ and $1.06 \AA$ for $\mathrm{Fe}, \mathrm{Co}, \mathrm{Ni}$, and $\mathrm{Si}$, in order to avoid overlap of the muffin-tin spheres (due to covalent bond-shortening within the TM silicide series, as we report below) during structure relaxations. This choice is reasonable in view of their respective atomic sizes. The cutoff energy for the interstitial plane-wave expansion is chosen to be 15.2 Ryd. ${ }^{28}$ A set of $10 \times 10 \times 1$ special $\mathbf{k}$ points is used for integrations over the Brillouin zone of the $(1 \times 1)$ surface cell. Except for the two central Si layers in the slab, all the $M$ and other $\mathrm{Si}$ atoms are relaxed until the calculated atomic force for each of them is smaller than $0.05 \mathrm{eV} / \AA$. Throughout this paper, formation energies are given per $(1 \times 1)$ cell, defined as

$$
E_{\text {form }}=\left(E_{\text {tot }}-\sum_{i} N_{i} \mu_{i}\right) / 2-\gamma_{\mathrm{Si}} A,
$$

where $E_{\text {tot }}, N_{i}$, and $\mu_{i}$ refer to the total energy per $(1 \times 1)$ unit cell with surface area $A$, the number of atoms of each chemical type in the cell, and their chemical potentials as calculated from the corresponding bulk materials. The factor 2 in the denominator is because the slab contains two equivalent surfaces due to the inversion symmetry. $\gamma_{\mathrm{Si}}=84 \mathrm{meV} / \AA^{2}$ is the surface energy of the clean, $p(2 \times 2)$-reconstructed $\mathrm{Si}(001)$ surface. We note that $E_{\text {form }}$ defined in this way contains the bulk heat of formation, as well as surface and interface contributions. The interface energy alone, which could serve as an indicator for adhesion of the films to the substrate, is not considered. The numerical accuracy of the present calculations is carefully checked by using higher cutoff energy and more $\mathbf{k}$ points. With these settings, the absolute values of $E_{\text {form }}$ are converged with respect to cutoff energy and k-point sampling to better than $0.1 \mathrm{eV}$. However, for the relative stability of structures with the same composition but different geometries and/or magnetic structures, we can give a much stricter error estimate, only several $\mathrm{meV}$, due to error cancellation since all numbers entering the energy difference are calculated with the same technical settings. The degree of spin polarization at the Fermi level is quantified from the spin-resolved density of states (DOS), which is calculated using a finer $\mathbf{k}$-point mesh of $16 \times 16 \times 1$ in conjunction with the tetrahedron method for Brillouin zone integration. We note that a more realistic assessment of spin injection at the interface would have to consider the match in Fermi velocities in the film and the substrate. For bulk magnets, a spin polarization including a suitable weighting with the Fermi velocity can be defined. ${ }^{29,30}$ However, in this work we retain the more widespread definition of the DOS.

\section{RESULTS AND DISCUSSION}

\section{A. Bulk phases of $\boldsymbol{M ~ S i}$}

Before studying the $M$ Si thin films on $\operatorname{Si}(001)$, we briefly discuss the bulk phases of the TM monosilicides $M \mathrm{Si}$ 


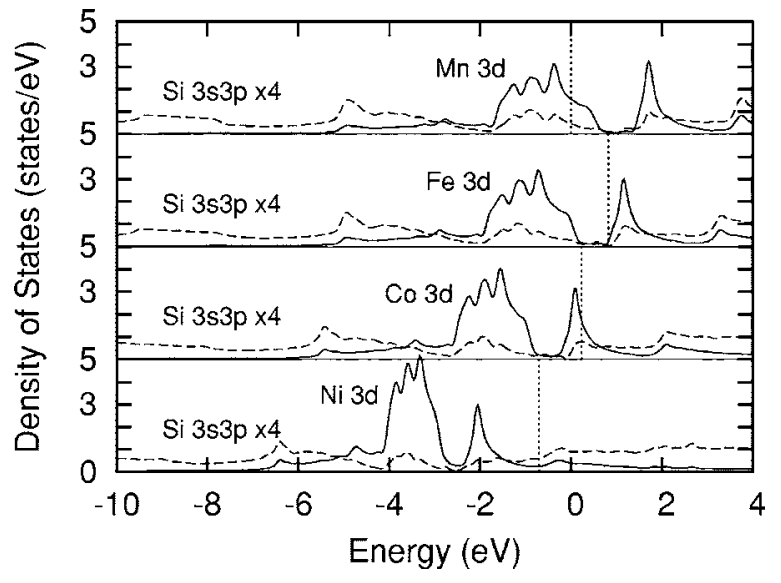

FIG. 2. Orbital-projected DOS of metastable CsCl-like bulk $M \mathrm{Si}(M=\mathrm{Mn}, \mathrm{Fe}, \mathrm{Co}, \mathrm{Ni})$ in the nonmagnetic state. The solid lines refer to the $M 3 d$ bands, which split into the lower-lying $t_{2 g}$ and the higher-lying $e_{g}$ bands. The dashed lines refer to the Si $3 s 3 p$ states (magnified four times for clarity). The Fermi level of MnSi (calculated to be $11.76 \mathrm{eV}$ ) is used as energy zero for all plots. The Fermi levels (vertical dotted lines) of $\mathrm{FeSi}, \mathrm{CoSi}$, and NiSi differ from that of $\mathrm{MnSi}$ by $0.81,0.23$, and $-0.69 \mathrm{eV}$, respectively. Obviously, the shapes of those DOS are similar, and the Fermi level shifts towards and strides over the $e_{g}$ states to accommodate more and more $d$ electrons as $M$ varies from $\mathrm{Mn}$ through $\mathrm{Fe}$ and $\mathrm{Co}$ to Ni. Note that, as $M$ varies from $\mathrm{Mn}$ to $\mathrm{Ni}$, the $M 3 d$ bands monotonously shift down toward the $\mathrm{Si} 3 s 3 p$ valence bands.

$(M=\mathrm{Mn}, \mathrm{Fe}, \mathrm{Co}, \mathrm{Ni})$. For all metal atoms discussed here, the monosilicides have the same bulk crystal structure, the $B 20$ structure, whose symmetry is characterized by the $P 2_{1} 3$ space group. ${ }^{31}$ Since the lattice constant of the cubic unit cell is around $4.5 \AA$ for all these compounds, they cannot be lattice matched with $\mathrm{Si}(001)$. However, the metastable $\mathrm{CsCl}$ phase calculated within DFT-GGA lies only slightly above the ground-state $P 2{ }_{1} 3$ structure in total energy, for $M=\mathrm{Mn}$, $\mathrm{Fe}, \mathrm{Co}$, and $\mathrm{Ni}$ by $0.25,0.04,0.42$, and $0.24 \mathrm{eV}$ per formula unit, respectively. Moreover, it follows from our GGA calculations that the equilibrium lattice constants for the metastable $\mathrm{CsCl}$ phases are 2.79, 2.77, 2.78, and $2.85 \AA$, respectively. They are almost half the calculated lattice constant of Si $(5.48 \AA)$, and thus the lattice mismatch with $\mathrm{Si}(001)$ is less than $2 \%$ for the $\mathrm{CsCl}$-like $\mathrm{MnSi}, \mathrm{FeSi}$, and $\mathrm{CoSi}$, and $4 \%$ for $\mathrm{NiSi}$. These results for $M \mathrm{Si}(M=\mathrm{Fe}, \mathrm{Co})$ agree well with the previous calculations by Moroni, Podloucky, and Hafner. ${ }^{8}$

We show in Fig. 2 the density of states of the CsCl-like $M$ Si calculated within GGA in the nonmagnetic (NM) state. The CsCl-like FeSi and NiSi have a low DOS at the Fermi level, which explains, within the framework of the Stoner model of magnetism, why we find them to be nonmagnetic. In contrast, the Fermi level of the CsCl-like MnSi lies at a falling shoulder of the $t_{2 g}$ DOS. In particular, the Fermi level of the CsCl-like CoSi lies at a steep slope of the $e_{g}$ DOS, which gives rise to Stoner FM instability. This has also been discussed by Profeta et al. ${ }^{11}$ Our calculations show that the FM ground state of CoSi has a spin moment of $0.63 \mu_{B} / \mathrm{Co}$ and a lower total energy than the NM state by $16 \mathrm{meV}$ per formula unit.

Since epitaxial growth of the CsCl-like FeSi and CoSi films on $\mathrm{Si}(111)$ has already been achieved by von Känel $e t$
TABLE I. Formation energies [in units of eV per $(1 \times 1)$ cell] of films in various structures depicted in Fig. 1, labeled (a)-(f), relative to the clean $\mathrm{Si}(001)$ surface and elemental bulk $M=\mathrm{Mn}, \mathrm{Fe}, \mathrm{Co}$, or Ni. Note that the values of $E_{\text {form }}$ in the $M=\mathrm{Mn}$ row are slightly different (by $0.03 \mathrm{eV}$ at most) from those of our previous calculations (Ref. 5) given in parentheses, due to different values of the muffin-tin radius of $\mathrm{Si}$ and the cutoff energy used.

\begin{tabular}{lcccccc}
\hline \hline$E_{\text {form }}$ & $\mathrm{a}$ & $\mathrm{b}$ & $\mathrm{c}$ & $\mathrm{d}$ & $\mathrm{e}$ & $\mathrm{f}$ \\
\hline $\mathrm{Mn}$ & 0.76 & 0.67 & 0.89 & 0.61 & -0.43 & -1.55 \\
& $(0.77)$ & $(0.68)$ & $(0.90)$ & $(0.62)$ & $(-0.40)$ & $(-1.53)$ \\
$\mathrm{Fe}$ & 1.11 & 0.67 & 0.93 & 0.01 & -1.71 & -3.78 \\
$\mathrm{Co}$ & 0.99 & 0.47 & 0.89 & -0.44 & -2.38 & -4.15 \\
$\mathrm{Ni}$ & 0.59 & 0.18 & 0.22 & -0.64 & -2.37 & -3.46 \\
\hline \hline
\end{tabular}

al., ${ }^{9,10}$ and given that CoSi has the highest energy difference for the metastable phase among the CsCl-like $M \mathrm{Si}$ $(M=\mathrm{Mn}, \mathrm{Fe}, \mathrm{Co}, \mathrm{Ni})$, we consider it likely that growth of the CsCl-like $M$ Si films on $\mathrm{Si}(001)$, and of the CsCl-like MnSi and NiSi films on $\mathrm{Si}(111)$, can be achieved as well.

\section{B. $M$ Si thin films on $\mathrm{Si}(001)$}

For various amounts of TM atoms deposited on $\mathrm{Si}(001)$, we perform calculations to investigate the stable binding sites or the (meta)stable atomic structure of films. As seen below, the preceding calculations for $\theta=0.5$ and $1 \mathrm{ML}$ are helpful to understand why the $M$ atoms prefer subsurface sites and the $\mathrm{Si}$ atoms sit in the topmost layer.

We start our calculations by considering a coverage of $\theta=0.5 \mathrm{ML}$ of metal atoms $M$, occupying either atomic sites on the surface [see Fig. 1(a)] or subsurface sites [see Fig. 1(b)] of $\mathrm{Si}(001)$. The results show that all metal adsorbates, $M=\mathrm{Mn}, \mathrm{Fe}, \mathrm{Co}$, and $\mathrm{Ni}$, are generally more stable at $\mathrm{Si}(001)$ subsurface than at surface sites, by about $0.1 \mathrm{eV}$ per $(1 \times 1)$ cell for $M=\mathrm{Mn}$, and more than $0.4 \mathrm{eV}$ for $M=\mathrm{Fe}, \mathrm{Co}$, or $\mathrm{Ni}$, as seen in Table I. The surface adatoms $M=\mathrm{Mn}, \mathrm{Fe}$, and Co have a sizable spin moment, and in Table II, the values within the atomic muffin-tin spheres are reported. The reduction of the spin magnetic moment of $M$ atoms on subsurface sites is due to the increased number of $M$-Si bonds. In particular, the magnetic moment of the subsurface Co atom is almost completely quenched. Moreover, we find $\mathrm{Ni}$ atoms to have vanishing magnetic moments both on the surface and at subsurface sites. Note that in these $M-\mathrm{Si}(M=\mathrm{Mn}, \mathrm{Fe}, \mathrm{Co})$

TABLE II. Spin magnetic moment (in units of $\mu_{B}$ ) of $M$ atoms within muffin-tin spheres for various structures depicted in Fig. 1, labeled (a)-(d). NM Ni case is omitted. Reported for (c) are both values for the surface and subsurface $M$ atoms, separated by a comma; and for (d) are the substitutional and interstitial $M$ atoms.

\begin{tabular}{lcccc}
\hline \hline$m$ & $\mathrm{a}$ & $\mathrm{b}$ & $\mathrm{c}$ & $\mathrm{d}$ \\
\hline $\mathrm{Mn}$ & 3.68 & 3.08 & $3.26,2.25$ & $2.16,1.65$ \\
$\mathrm{Fe}$ & 2.35 & 2.09 & $2.45,1.94$ & $0.11,0.05$ \\
$\mathrm{Co}$ & 0.95 & 0.03 & $0.45,-0.07$ & $0.41,0.35$ \\
\hline \hline
\end{tabular}



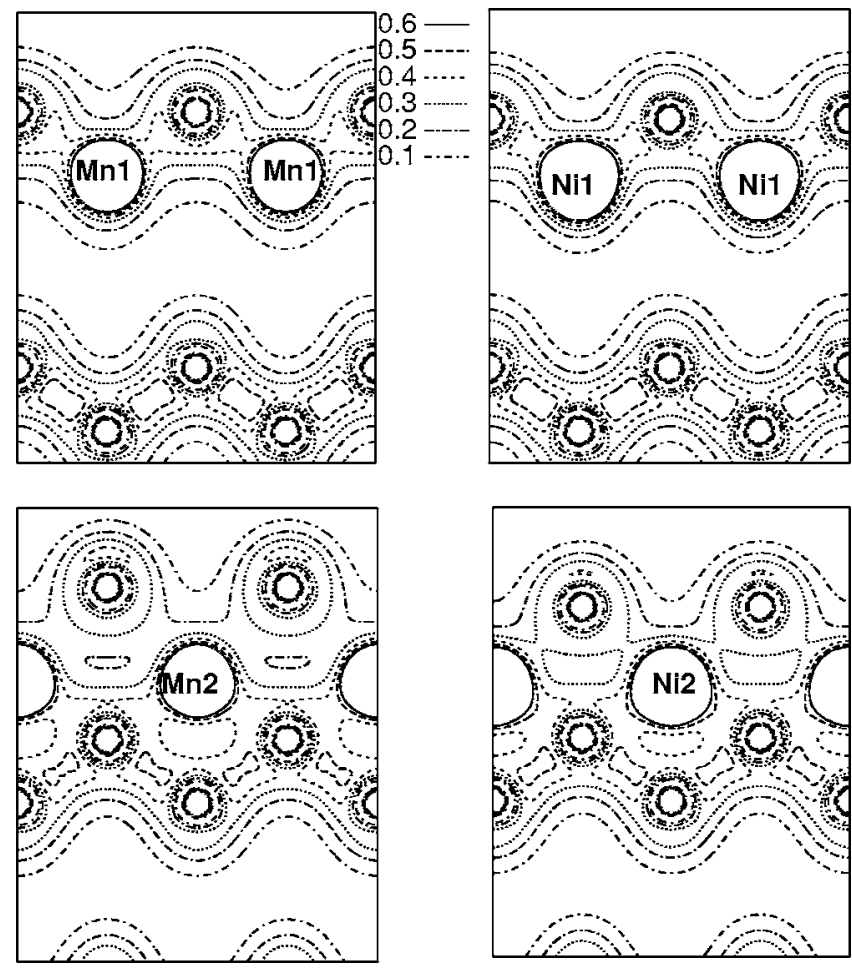

FIG. 3. Valence charge density in the $(1 \overline{1} 0)$ plane for $1 \mathrm{ML}$ Si-capped silicide films, $\mathrm{Si}-M / \mathrm{Si}(001)[M=\mathrm{Mn}$ (left panels) or $\mathrm{Ni}$ (right panels), see Fig. 1(d)]. The cuts are chosen to contain the substitutional-M1 and $\mathrm{Si}$ (upper row), or the interstitial-M2 and $\mathrm{Si}$ atoms (lower row). Contour lines from 0.1 to $0.6 e / \AA^{3}$ in steps of $0.1 \mathrm{e} / \AA^{3}$ are shown. The $\mathrm{Mn}-\mathrm{Si}$ and $\mathrm{Ni}-\mathrm{Si}$ bonds have a covalent charge density as high as $0.4 e / \AA^{3}$, similar to the Si-Si bonds with $0.5 \mathrm{e} / \AA^{3}$.

systems, spin moments are also induced on the $\mathrm{Si}$ atoms adjacent to $M$, albeit smaller than $0.1 \mu_{B}$.

Secondly, we compare two possible atomic structures for 1 ML coverage, the 1 ML- $M$ surface mixed layer [see Fig. 1(c)] and the layered Si-M film [see Fig. 1(d)]. Our results show that the latter is energetically more favorable than the former, by about $0.3 \mathrm{eV}$ per $(1 \times 1)$ cell for $M=\mathrm{Mn}$ and around $1.0 \mathrm{eV}$ for $M=\mathrm{Fe}, \mathrm{Co}$, or Ni. Next, we analyze the chemical bonding in these systems. We start by noting that $M$ (=Mn, Fe, Co, or Ni) and $\mathrm{Si}$ have almost identical electronegativity of 1.6 or 1.7 , and hence form strong covalent bonds. From Fig. 3, we see that the $M$-Si bonds have similar covalent charge density as the $\mathrm{Si}-\mathrm{Si}$ bonds. Moreover, for all relaxed structures of the $\mathrm{Si}-M / \mathrm{Si}(001)(M=\mathrm{Mn}, \mathrm{Fe}, \mathrm{Co})$ films, we find that both the substitutional $M$ (named $M 1$ ) and the interstitial $M$ (named $M 2$ ) each have four $M$-Si bonds which are shorter, by $0.13 \AA$ at least, than the sum of the $M$ and $\mathrm{Si}$ atomic radii, due to covalent bond contraction. NiSi is an exception to this general trend; in $\mathrm{Si}-\mathrm{Ni} / \mathrm{Si}(001)$ the substitutional $\mathrm{Ni1}$ has four shrunk $\mathrm{Ni}-\mathrm{Si}$ bonds which are contracted by $0.08 \AA$, and the interstitial $\mathrm{Ni} 2$ has only two short Ni-Si bonds, contracted by $0.18 \AA$. This exceptional behavior, both the smaller Ni1-Si bond-shortening and the reduced number of short Ni2-Si bonds, can be understood by considering that the number of empty $3 d$ orbitals available for bonding with $\mathrm{Si}$ decreases in the TM series from $\mathrm{Mn}$ to Ni.
Note that the transition metal atoms are sevenfold coordinated to $\mathrm{Si}$ in the natural bulk silicides $M \mathrm{Si}$, and eightfold coordinated in $\mathrm{M} \mathrm{Si}_{2}$. Thus, the subsurface TM layer capped by a $\mathrm{Si}$ layer in the $\mathrm{Si}-\mathrm{M} / \mathrm{Si}(001)$ films optimizes the surface covalent bonding structure, since it allows for the optimum fourfold coordination of the capping $\mathrm{Si}$ atoms, while simultaneously increasing the coordination of the $M$ atoms (compared to on surface adsorption). The Si termination of the CsCl-like FeSi/Si(111) film surface has been previously verified both experimentally and theoretically. ${ }^{32}$ Moreover, the $\mathrm{Si}$ capping layer, due to the doubled atomic density as compared with the $\mathrm{Si}(001)$ substrate, displays strong buckling, $0.43,0.57,0.47$, and $0.21 \AA$ in the $\mathrm{Si}-M / \mathrm{Si}(001)$ film with $M=\mathrm{Mn}, \mathrm{Fe}, \mathrm{Co}$, or $\mathrm{Ni}$, respectively.

Since the layered Si- $M$ film has turned out to be energetically most favorable from the above calculations, we employ the same atomic structure to multilayered $\mathrm{Si}-M[n(\mathrm{Si}-\mathrm{Mn})]$ films, i.e., to the $\mathrm{CsCl}$-like $M \mathrm{Si}$ films with $\mathrm{Si}$ termination, as depicted in Figs. 1(e) and 1(f). As seen in columns (d), (e), and (f) of Table I, the formation energy $E_{\text {form }}$, defined according to Eq. (1), decreases monotonously with increasing film thickness for all CsCl-like $M \mathrm{Si}$ films. This decrease is a consequence of the heat of formation released for each formula unit of $M \mathrm{Si}$ formed from the elements. The onset of negative $E_{\text {form }}$ at $\theta \approx 2 \mathrm{ML} \mathrm{Mn} \mathrm{or} 1 \mathrm{ML} M(M=\mathrm{Fe}, \mathrm{Co}, \mathrm{Ni})$ indicates that the films are stable against decomposition into the clean $\mathrm{Si}(001)$ surface and elemental bulk $M$.

Moreover, the thermodynamic stability of the $M$ Si films increases as $M$ varies from $\mathrm{Mn}$ through $\mathrm{Fe}, \mathrm{Co}$, to $\mathrm{Ni}$ at $\theta<2 \mathrm{ML}$. We attribute this finding to the increasing $M-\mathrm{Si}$ bond strength: Note that $E_{\text {form }}$ is calculated with reference to the clean $\mathrm{Si}(001)$ surface and elemental TM bulk [see Eq. (1)]. Both GGA calculations and experimental measurements agree that the cohesive energies of $\mathrm{Fe}, \mathrm{Co}$, and $\mathrm{Ni}$ are very similar, and higher than that of Mn by about $1 \mathrm{eV}^{26}$ Therefore the decreasing $E_{\text {form }}$ of the $M$ Si films at $\theta<2 \mathrm{ML}$ as $M$ varies from $\mathrm{Mn}$ to the later TMs indicates that the binding energy of the $M$ atoms on $\mathrm{Si}(001)$ increases more strongly so as to overcompensate the rise in the removal energy of an $M$ atom from its bulk reservoir upon variation of $M$ from $\mathrm{Mn}$ to the later TMs. Hence, the strength of the $M$-Si bonds must increase accordingly. This trend can be understood by observing that the $M 3 d$ bands increasingly come into resonance with the $\mathrm{Si} 3 s 3 p$ valence bands due to decreasing energy separation between them (see Fig. 2), because the $M 3 d$ level shifts down towards the $\mathrm{Si} 3 s 3 p$ level as the atomic number of the transition metal increases. However, the trend is reversed for the NiSi film at $\theta=2 \mathrm{ML}$ [see column (e) in Table I]. For thicker $M$ Si films, the order of thermodynamic stability, quoted from low to high, changes to $M=\mathrm{Mn}, \mathrm{Ni}$, $\mathrm{Fe}, \mathrm{Co}$ at $\theta=3 \mathrm{ML}$ [see column (f) in Table I]. The anomaly in the NiSi case can be explained in terms of $M 3 d$ orbital occupation. Since Ni has the fewest empty $3 d$ orbitals available for bonding with $\mathrm{Si}$, the Ni atoms in the NiSi film (except for the interfacial $\mathrm{Ni}$ ) being eightfold coordinated to $\mathrm{Si}$ become oversaturated. The oversaturation for eightfold $\mathrm{Si}$ coordination of $\mathrm{Ni}$ is also reflected by the increased lattice constant of the CsCl-like NiSi [compared with $M \mathrm{Si}$ $(M=\mathrm{Mn}, \mathrm{Fe}, \mathrm{Co})$ as seen in Sec. III A]. This interpretation is corroborated by the experimental observation that the lattice 
constant of the eightfold coordinated $\mathrm{NiSi}_{2}$ is larger than that of $\mathrm{CoSi}_{2}$.

The above results are helpful to understand three experimental observations. First, preadsorbed Co has been found to improve the quality of $\mathrm{Fe}$ films grown on $\mathrm{Si}(001) .{ }^{33}$ Our calculations show that $\mathrm{Co}-\mathrm{Si}$ bonds are stronger than $\mathrm{Fe}-\mathrm{Si}$ bonds; hence the improved film quality can be explained by a CoSi layer forming at the interface which prevents interdiffusion between the Fe overlayer and the Si substrate. Moreover, we can predict that $\mathrm{Ni}$ cannot be used for this purpose, because the highly $\mathrm{Si}$-coordinated $\mathrm{Ni}$ silicide is thermodynamically less stable than $\mathrm{Fe}$ silicide, as we reported above. Hence, we conclude from our calculations that $\mathrm{Ni}$ is unsuitable for a barrier layer to suppress the intermixing between $\mathrm{Fe}$ and $\mathrm{Si}$. Secondly, the trends in bond strength revealed by our calculations help to explain the structure of Heusler alloys with the chemical composition $M_{2} \mathrm{MnSi}$ $(M=\mathrm{Fe}, \mathrm{Co}, \mathrm{Ni})$, or more generally $X_{2} Y Z,{ }^{2-4}$ in which $X, Y$, and $Z$ have a similar electronegativity and $Y$ possesses a robust magnetic moment. In these materials, so-called full Heusler alloys, which can be considered as a (111) stacking of layers with the sequence $Z-X-Y-X-Z-X-Y-X-Z \cdots$, it is always the element $X$ capable of making stronger bonds to $Z$ which occurs in the layers adjacent to $Z$, while the more weakly bonding element $Y$ has $Z$ only as its second neighbors. Together with knowledge of the energetic positions of the atomic levels of the $X, Y$, and $Z$ atoms, and thus of their relative bond strengths, this rule can be used as heuristics in the search for new Heusler alloys (some of which may be half-metallic FMs), somewhat similar in spirit to the "band gap engineering" done in semiconductor physics. Thirdly, on the basis of our results, we can explain the observed site selectivity ${ }^{34}$ for substitution of other TMs in the Heusler alloy $\mathrm{Fe}_{2}^{A} \mathrm{Fe}^{B} \mathrm{Si}$ : The TMs to the right of $\mathrm{Fe}$ in the periodic table, $\mathrm{Co}$ and $\mathrm{Ni}$, making stronger bonds to $\mathrm{Si}$ than $\mathrm{Fe}$ itself, substitute for $\mathrm{Fe}^{A}$ to form new stronger bonds with four $\mathrm{Si}$ neighbors. The earlier TMs Ti, V, Cr, Mn, however, substitute for $\mathrm{Fe}^{B}$, thus preserving the stronger $\mathrm{Fe}^{A}-\mathrm{Si}$ bonds.

Next we turn to the magnetism of the $M S i$ thin films on $\mathrm{Si}(001)[n(\mathrm{Si}-M) / \mathrm{Si}(001)]$. As a general trend in the pseudomorphic $(\mathrm{Si}-M) / \mathrm{Si}(001)$ films [see Fig. 1(d)], we find that the substitutional $M 1$ (see Fig. 3) has a little larger spin moment (e.g., $2.16 \mu_{B} / \mathrm{Mn} 1$ ) than the interstitial $M 2$ (e.g., $\left.1.65 \mu_{B} / \mathrm{Mn} 2\right)$, as seen in Table II. This can be partly ascribed to the number of $M$-Si bond being fewer by one for $M 1$ (sixfold coordination) than $M 2$ (sevenfold coordination). First, we describe in more detail the results for MnSi films. The $(\mathrm{Si}-\mathrm{Mn}) / \mathrm{Si}(001)$ film is found from our calculations to be a ferromagnetic metal with a sizable spin moment, in which the $\mathrm{Si}$ atoms mediate the FM Mn-Mn coupling via hybridization between the $\mathrm{Si} 3 s 3 p$ and $\mathrm{Mn} 3 d$ itinerant electrons. A vital role is played by the capping $\mathrm{Si}$ atoms; in their absence the bare $\mathrm{Mn}$ film on $\mathrm{Si}(001)$ is found to be antiferromagnetic (AFM). ${ }^{5}$ For the $2(\mathrm{Si}-\mathrm{Mn}) / \mathrm{Si}(001)$ film, our calculations also predict a FM metallic ground state. The $3(\mathrm{Si}-\mathrm{Mn}) / \mathrm{Si}(001)$ film is found to be ferrimagnetic with FM (ferrimagnetic) intralayer (interlayer) coupling, as seen in Tables III and IV. The middle Mn layer has a small spin moment of $-0.14 \mu_{B} / \mathrm{Mn}$ antiparallel to the larger one of
TABLE III. Spin magnetic moment (in units of $\mu_{B}$ ) of atoms averaged over one layer [from interface layer (left) to surface layer (right)] of the $M \mathrm{Si}$ thin films on $\mathrm{Si}(001)$ [see Figs. 1(d)-1(f)] in their respective magnetic ground states. Note that the $\mathrm{FeSi} / \mathrm{Si}(001)$ films are nonmagnetic, as discussed in the text. The nonmagnetic $\mathrm{NiSi} / \mathrm{Si}(001)$ films are omitted.

\begin{tabular}{lcccccc}
\hline \hline & $M$ & $\mathrm{Si}$ & $M$ & $\mathrm{Si}$ & $M$ & $\mathrm{Si}$ \\
\hline Si-Mn & 1.90 & -0.05 & & & & \\
Si-Fe & 0.08 & -0.01 & & & & \\
Si-Co & 0.38 & 0.02 & & & & \\
2(Si-Mn) & 1.90 & -0.07 & 1.11 & 0.02 & & \\
2(Si-Fe) & 0.38 & -0.01 & 0.06 & 0.01 & & \\
2(Si-Co) & 0.16 & -0.01 & 0.55 & -0 & & \\
3(Si-Mn) & 1.74 & -0.03 & -0.14 & 0.03 & -1.07 & -0.04 \\
3(Si-Fe) & 0.31 & -0.01 & 0.01 & -0 & 0.01 & +0 \\
3(Si-Co) & 0.38 & -0.01 & 0.56 & -0.01 & 0.63 & -0.01 \\
\hline \hline
\end{tabular}

$1.74 \mu_{B} / \mathrm{Mn}$ in the interfacial $\mathrm{Mn}$ layer. It mediates a superexchange ferrimagnetic coupling between the interfacial and subsurface Mn layers. Note that the interlayer magnetic coupling is weak in the $n(\mathrm{Si}-\mathrm{Mn}) / \mathrm{Si}(001)$ thin films, e.g., the energy cost for flipping the magnetic moments of one layer, i.e., going from FM to AFM ordering between layers, is 8 and $10 \mathrm{meV} / \mathrm{Mn}$ in the $2(\mathrm{Si}-\mathrm{Mn}) / \mathrm{Si}(001)$ and $3(\mathrm{Si}-\mathrm{Mn}) / \mathrm{Si}(001)$ films, respectively. However, the FM intralayer coupling is rather strong, as is evident from the energy cost for flipping one of the two magnetic moments per layer in the unit cell, i.e., going from FM to AFM ordering within the layers, which we calculate to be $70-80 \mathrm{meV} / \mathrm{Mn}$. Moreover, the various magnetic MnSi films we studied have a spin polarization of carriers at the Fermi level in the range of 30-50\%. These results imply that the ultrathin MnSi film on $\mathrm{Si}(001)$ is a candidate for magnetoelectronic materials.

For the $(\mathrm{Si}-\mathrm{Fe}) / \mathrm{Si}(001)$ film, our calculations find the AFM state to be unstable and to converge to the FM ground state (with a very small spin moment, as seen in Tables III and IV). However, the FM state and the NM state are energetically degenerate, as seen in Table IV. Similarly, the FM state of the $2(\mathrm{Si}-\mathrm{Fe}) / \mathrm{Si}(001)$ and $3(\mathrm{Si}-\mathrm{Fe}) / \mathrm{Si}(001)$ films has a small spin moment and almost the same energy as the NM state, the energy difference being less than $5 \mathrm{meV} / \mathrm{Fe}$.

TABLE IV. Total-energy difference (in units of meV per $M$ atom) of the $n(\mathrm{Si}-M) / \mathrm{Si}(001) \quad(n=1,2,3 ; M=\mathrm{Mn}, \mathrm{Fe}, \mathrm{Co})$ thin films among the ferromagnetic (FM), antiferromagnetic (AFM, either intralayered or interlayered AFM marked with superscript $i$ or $o$ ), and nonmagnetic (NM) states.

\begin{tabular}{|c|c|c|c|c|c|c|c|c|c|}
\hline \multirow[b]{2}{*}{$n$} & \multicolumn{3}{|c|}{$n(\mathrm{Si}-\mathrm{Mn})$} & \multicolumn{3}{|c|}{$n(\mathrm{Si}-\mathrm{Fe})$} & \multicolumn{3}{|c|}{$n(\mathrm{Si}-\mathrm{Co})$} \\
\hline & 1 & 2 & 3 & 1 & 2 & 3 & 1 & 2 & 3 \\
\hline FM & 0 & 0 & 10 & 0 & 0 & 0 & 0 & 0 & 0 \\
\hline AFM & $71^{i}$ & $8^{\circ}$ & $0^{o}$ & FM & 0 & 0 & $\mathrm{NM}$ & 0 & $10^{\circ}$ \\
\hline NM & 350 & 188 & 80 & 0 & 5 & 0 & 15 & 17 & 28 \\
\hline
\end{tabular}




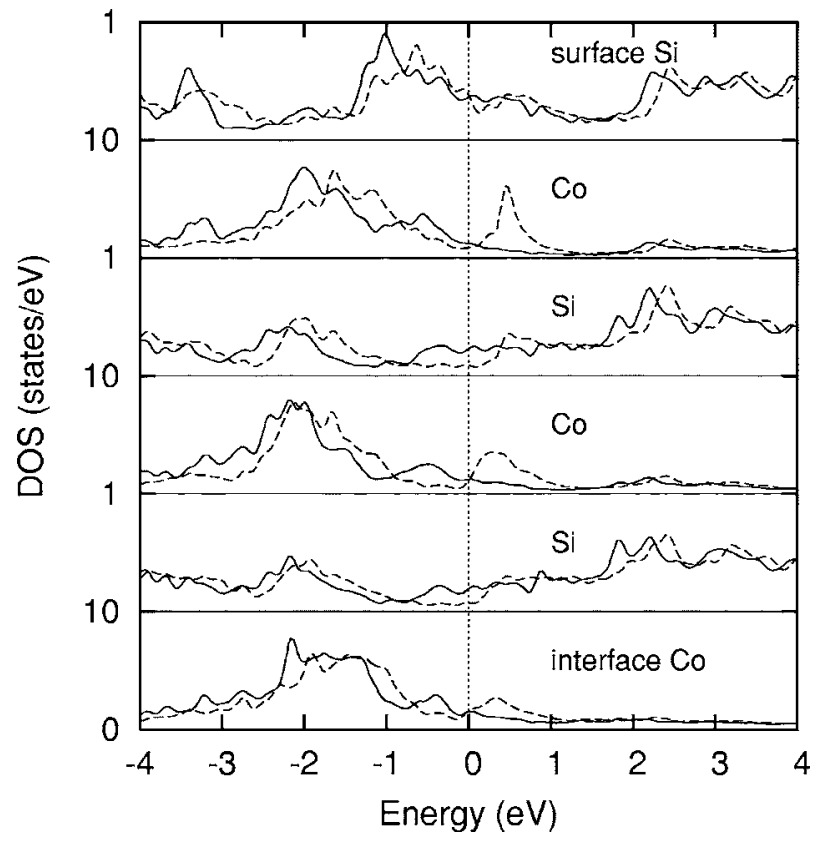

FIG. 4. The layer-resolved DOS of the FM 3(Si-Co)/Si(001) film. The layers are shown from surface (top) to interface (bottom) for the atomic structure depicted in Fig. 1(f). Full lines show the majority spin, dashed lines the minority spin component.

Therefore we conclude that the $\mathrm{FeSi} / \mathrm{Si}(001)$ films are NM, similar to the CsCl-like FeSi bulk, as discussed in Sec. III A. The $\mathrm{NiSi} / \mathrm{Si}(001)$ film is also NM, as evidenced by our computational results that both FM and AFM states converge to the NM ground state.

In strong contrast to the $\mathrm{NM} \mathrm{FeSi}$ and $\mathrm{NiSi}$ films on $\mathrm{Si}(001)$, the CoSi films on $\mathrm{Si}(001)$ have a $\mathrm{FM}$ ground state. This is evident from the magnetic moments reported in Table III and from the energetics reported in Table IV. In our calculations, a hypothetical AFM state of ( $\mathrm{Si}-\mathrm{Co}) / \mathrm{Si}(001)$ converges to a NM state which is, however, higher in total energy than the FM ground state by $15 \mathrm{meV} / \mathrm{Co}$. The $3(\mathrm{Si}-\mathrm{Co}) / \mathrm{Si}(001)$ film is also FM with a sizable spin moment in the middle layer (well comparable with the bulk value of $0.63 \mu_{B} / \mathrm{Co}$ ), unlike the ferrimagnetic $3(\mathrm{Si}-\mathrm{Mn}) / \mathrm{Si}(001)$ film. For 3(Si-Co)/Si(001), the layered AFM state is higher in total energy than the FM ground state by $10 \mathrm{meV} / \mathrm{Co}$. Moreover, our calculations find an increasing energy difference between the FM ground state and the NM state: 15,17 , and $28 \mathrm{meV} / \mathrm{Co}$ in the (Si-Co), 2(Si-Co), and $3(\mathrm{Si}-\mathrm{Co}) / \mathrm{Si}(001)$ films, respectively. We show in Fig. 4 the layer-resolved DOS of the FM 3(Si-Co) overlayers. The Fermi level is found to be close to a minimum of the Co $3 d$ DOS. Obviously the high DOS at the Fermi level seen in Fig. 2 for hypothetical NM CoSi has transformed into a minimum of the FM DOS due to exchange splitting. For this reason, the FM state is stable. Analyzing the DOS projected onto each $\mathrm{Si}$ overlayer, we find a considerable spin polarization of carriers at the Fermi level in the interior and near-interface $\mathrm{Si}$ overlayers, although those $\mathrm{Si}$ atoms themselves possess only a tiny induced spin moment.

These results suggest that the CsCl-like $\mathrm{CoSi} / \mathrm{Si}(001)$ films are interesting materials systems, having a high ther- modynamic stability among the $M \mathrm{Si} / \mathrm{Si}(001)$ films (see Table I) and a FM metallic ground state. Since the epitaxial growth of the CsCl-like CoSi film on $\mathrm{Si}(111)$ has already been achieved, ${ }^{10}$ attempting to grow a $\mathrm{CoSi} / \mathrm{Si}(001)$ film may be worth the experimental effort. Moreover, the predicted ferromagnetism of the $\mathrm{CsCl}$-like $\mathrm{CoSi}$ calls for experimental investigations. ${ }^{35}$

\section{C. $M_{2} \mathrm{MnSi}$ thin films on $\mathrm{Si}(001)$}

In this section, we study films of the Heusler alloys $M_{2} \mathrm{MnSi}(M=\mathrm{Fe}, \mathrm{Co}, \mathrm{Ni})$, which one can think of as being formed by partial $\mathrm{Mn}$ substitution for $\mathrm{Si}$ in the $\mathrm{CsCl}$-like $M \mathrm{Si}$ films (see Fig. 1) described so far. In particular, the Heusler alloy $\mathrm{Co}_{2} \mathrm{MnSi}$ is of interest here, since its bulk FM half-metallicity predicted by band calculations attracts much attention both from the experimental ${ }^{36-38}$ and theoretical ${ }^{2-4,39,40}$ side. Bulk $\mathrm{Fe}_{2} \mathrm{MnSi}$, in an ideal FM state, is also predicted by band calculations to be half-metallic. ${ }^{41}$ However, calculations allowing for noncollinear alignment of the magnetic moments have found that, in the ground state, the Mn magnetic moments are canted with respect to the direction of the $\mathrm{Fe}$ magnetic moments ${ }^{42}$ which leads to partial compensation of the magnetic moments along the [111] axis. The hypothetical compound $\mathrm{Ni}_{2} \mathrm{MnSi}$, which has not been synthesized so far to our knowledge, is shown by our calculations not to be half metallic. For the $\mathrm{Co}_{2} \mathrm{MnSi}(001)$ surface, it has been shown recently by means of DFT calculations ${ }^{21}$ that the termination by a Mn-Si crystal plane is thermodynamically stable, but a purely $\mathrm{Mn}$ - or purely Si-terminated surface can be stable as well under very $\mathrm{Mn}$-rich or under very Si-rich conditions, respectively.

The goal of this work is to investigate how finite-size effects and epitaxial strain in very thin films affect the magnetic properties. The latter effect, lowering the crystallographic symmetry, could possibly change the half-metallicity of $\mathrm{Co}_{2} \mathrm{MnSi}$ and $\mathrm{Fe}_{2} \mathrm{MnSi}$ films. In particular, we investigate how possible surface and interface electronic states affect the electronic and magnetic properties of the films. To this end, we perform systematic studies as a function of film thickness. Moreover, we consider various possibilities for the surface termination of the films, either Si surface termination [see Figs. 5(a) and 5(b)] or MnSi termination [see Figs. 5(c)-5(e)]. Note that the $M=\mathrm{Fe}, \mathrm{Co}$, or Ni termination is energetically unfavorable for reasons discussed in the previous section, and thus disregarded in this work. In addition to the two types of surface termination, two types of interfaces are studied, namely, the $M / \mathrm{Si}$ interface (see Fig. 5) and the $\mathrm{MnSi} / \mathrm{Si}$ interface. The latter is characterized by extra $\mathrm{Mn}$ atoms occupying the interstitial sites of the interfacial $\mathrm{Si}$ layer (not shown). First, we study the $M_{2} \mathrm{MnSi} / \mathrm{Si}(001)$ films with $\mathrm{Si}$ termination and $M / \mathrm{Si}$ interface. Secondly, we deal with films with $\mathrm{MnSi}$ termination and $\mathrm{M} / \mathrm{Si}$ interface. Thirdly, we discuss also the $\mathrm{MnSi} / \mathrm{Si}$ interface, but restrict ourselves to $\mathrm{Co}_{2} \mathrm{MnSi} / \mathrm{Si}(001)$ films, since they are thermodynamically stable and have a robust FM metallic ground state, as seen below, and hence are most relevant.

\section{1. $M_{2} \mathrm{MnSi} / \mathrm{Si}(001)$ : Si termination and $\mathrm{M} / \mathrm{Si}$ interface}

In this section, we use the terms two-layered [see Fig. 5(a)] and three-layered [see Fig. 5(b)] Heusler alloy films, 
(a)

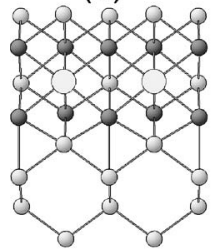

(b)

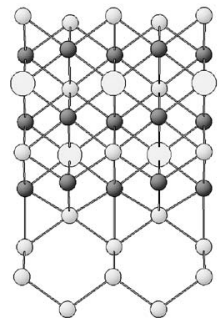

(c)

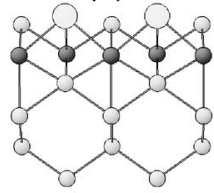

(d)

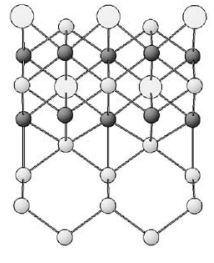

(e)

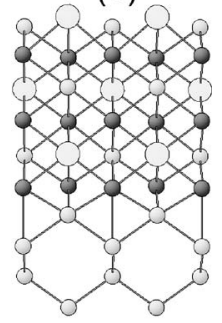

[001]

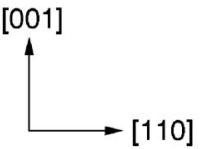

FIG. 5. Side view of the Si-terminated two-layered (a) and three-layered (b) Heusler alloy $M_{2} \mathrm{MnSi}(M=\mathrm{Fe}, \mathrm{Co}, \mathrm{Ni})$ films on $\mathrm{Si}(001)$ with $\mathrm{M} / \mathrm{Si}$ interface, and of the MnSi-terminated onelayered (c), two-layered (d), and three-layered (e) $M_{2} \mathrm{MnSi}$ films. Black balls represent $M$, gray balls $\mathrm{Si}$, and large white balls $\mathrm{Mn}$ atoms. The bonds shorter than $2.65 \AA$ are shown. according to the film thickness measured in repetition periods of the atomic superstructure of the alloy. First, we discuss the results for the two- and three-layered films, focussing on magnetic ordering. Independent on composition, we find for all the two-layered $M_{2} \mathrm{MnSi}$ films a metallic ground state with FM coupling both in the Mn sublattice and between the $\mathrm{Mn}$ and $M$ sublattices $(M=\mathrm{Fe}, \mathrm{Co}, \mathrm{Ni})$. For $\mathrm{Fe}_{2} \mathrm{MnSi}$, AFM ordering among the magnetic moments of $\mathrm{Fe}$ and $\mathrm{Mn}$ is metastable, but higher than the FM state in total energy by $20 \mathrm{meV}$ per $(1 \times 1)$ cell. For the $\mathrm{Co}_{2} \mathrm{MnSi}$ and $\mathrm{Ni}_{2} \mathrm{MnSi}$ films, however, AFM ordering of the magnetic moments of the Co and $\mathrm{Mn}$ (or of $\mathrm{Ni}$ and $\mathrm{Mn}$, respectively) is found to be unstable, and the calculations converge to the FM ground state. Moreover, our results show that the effective Mn-Mn FM coupling is strong, since the calculated energy cost to flip a Mn-Mn spin pair from parallel to antiparallel orientation is as high as $73 \mathrm{meV} / \mathrm{Mn}$ in $\mathrm{Fe}_{2} \mathrm{MnSi}$, $216 \mathrm{meV} / \mathrm{Mn}$ in $\mathrm{Co}_{2} \mathrm{MnSi}$, and $80 \mathrm{meV} / \mathrm{Mn}$ in $\mathrm{Ni}_{2} \mathrm{MnSi}$. Note that in the two-layered $\mathrm{M}_{2} \mathrm{MnSi}$ films, the Mn atoms have the same environment as in the bulk. Therefore it is not surprising that the calculated Mn-Mn coupling strengths approximately scale with the measured FM Curie temperatures

TABLE V. The layer-resolved (counted from the substrate to the surface) atomic spin moments (in units of $\mu_{B}$ ) of the Si-terminated two-layered $(2 L)$ and $3 \mathrm{~L} \mathrm{M}_{2} \mathrm{MnSi} / \mathrm{Si}(001)$ films and of the MnSi-terminated $1 L, 2 L$, and $3 L \mathrm{M}_{2} \mathrm{MnSi} / \mathrm{Si}(001)$ films (cf. Fig. 5). All films have a $M / \mathrm{Si}$ interface. Shown in the last three rows are the calculated atomic spin moments of $\mathrm{Fe}_{2} \mathrm{MnSi}$ and $\mathrm{Co}_{2} \mathrm{MnSi}$ at the experimental lattice constant and of $\mathrm{Ni}_{2} \mathrm{MnSi}$ (not yet synthesized) at the GGA optimized lattice constant.

\begin{tabular}{|c|c|c|c|c|c|c|c|c|c|c|c|}
\hline Si-term. & $M$ & $\mathrm{Si} 4$ & $\mathrm{Si} 3$ & $\mathrm{Si} 2$ & Sil & $M$ & $\mathrm{MnSi}$ & $M$ & $\mathrm{MnSi}$ & $M$ & $\mathrm{Si}$ \\
\hline \multirow[t]{3}{*}{$2 L$} & $\mathrm{Fe}$ & 0.003 & -0.001 & 0.015 & -0.007 & & & 0.61 & $2.24 /-0.02$ & 0.36 & 0.14 \\
\hline & Co & 0.005 & 0.005 & 0.013 & -0.005 & & & 0.55 & $2.77 /-0.04$ & 0.70 & 0.01 \\
\hline & $\mathrm{Ni}$ & -0.002 & -0.006 & -0.002 & -0.009 & & & 0.14 & $3.06 /-0.04$ & 0.13 & -0.02 \\
\hline \multirow[t]{3}{*}{$3 L$} & $\mathrm{Fe}$ & 0.001 & -0 & 0.011 & -0 & 0.20 & $2.20 /-0.01$ & 0.21 & $2.31 /-0.01$ & 0.35 & 0.08 \\
\hline & Co & 0.004 & 0.005 & 0.007 & -0.006 & 0.53 & $2.74 /-0.04$ & 0.95 & $2.72 /-0.04$ & 0.71 & -0.01 \\
\hline & $\mathrm{Ni}$ & 0 & -0.003 & 0.004 & -0.007 & 0.16 & $3.03 /-0.03$ & 0.28 & $3.14 /-0.04$ & 0.12 & -0.02 \\
\hline MnSi-term. & $M$ & $\mathrm{Si} 4$ & $\mathrm{Si} 3$ & $\mathrm{Si} 2$ & Sil & $M$ & $\mathrm{MnSi}$ & $M$ & $\mathrm{MnSi}$ & $M$ & $\mathrm{MnSi}$ \\
\hline \multirow[t]{3}{*}{$1 L$} & $\mathrm{Fe}$ & 0.001 & 0 & 0.010 & -0.005 & & & & & 0.84 & $3.42 /-0.10$ \\
\hline & Co & 0 & 0.002 & 0.005 & -0 & & & & & 0.42 & $3.56 /-0.10$ \\
\hline & $\mathrm{Ni}$ & -0.001 & -0.002 & 0.001 & -0.007 & & & & & 0.02 & $3.58 /-0.10$ \\
\hline \multirow[t]{3}{*}{$2 L$} & $\mathrm{Fe}$ & 0.002 & -0.002 & 0.018 & -0.010 & & & 0.64 & $2.09 /-0.02$ & -0.06 & $3.45 /-0.10$ \\
\hline & Co & 0.005 & 0.004 & 0.013 & -0.011 & & & 0.54 & $2.65 /-0.05$ & 0.82 & $3.52 /-0.12$ \\
\hline & $\mathrm{Ni}$ & -0.001 & -0.004 & -0.001 & -0.005 & & & 0.18 & $3.05 /-0.03$ & 0.23 & $3.63 /-0.10$ \\
\hline \multirow[t]{3}{*}{$3 L$} & $\mathrm{Fe}$ & 0.001 & -0.002 & 0.011 & -0.007 & 0.47 & $2.21 /-0.02$ & 0.01 & $2.17 /-0$ & 0.18 & $3.50 /-0.11$ \\
\hline & Co & 0.004 & 0.003 & 0.008 & -0.013 & 0.52 & $2.70 /-0.04$ & 0.99 & $2.73 /-0.04$ & 0.86 & $3.53 /-0.11$ \\
\hline & $\mathrm{Ni}$ & 0 & -0.003 & 0.002 & -0.006 & 0.15 & $3.06 /-0.03$ & 0.29 & $3.12 /-0.04$ & 0.17 & $3.61 /-0.11$ \\
\hline Bulk $M_{2} \mathrm{MnSi}$ & $M$ & Mn & $\mathrm{Si}$ & & & & & & & & \\
\hline $\mathrm{Fe}_{2} \mathrm{MnSi}$ & 0.083 & 2.769 & -0 & & & & & & & & \\
\hline $\mathrm{Co}_{2} \mathrm{MnSi}$ & 0.987 & 3.013 & -0.039 & & & & & & & & \\
\hline $\mathrm{Ni}_{2} \mathrm{MnSi}$ & 0.290 & 3.330 & -0.028 & & & & & & & & \\
\hline
\end{tabular}



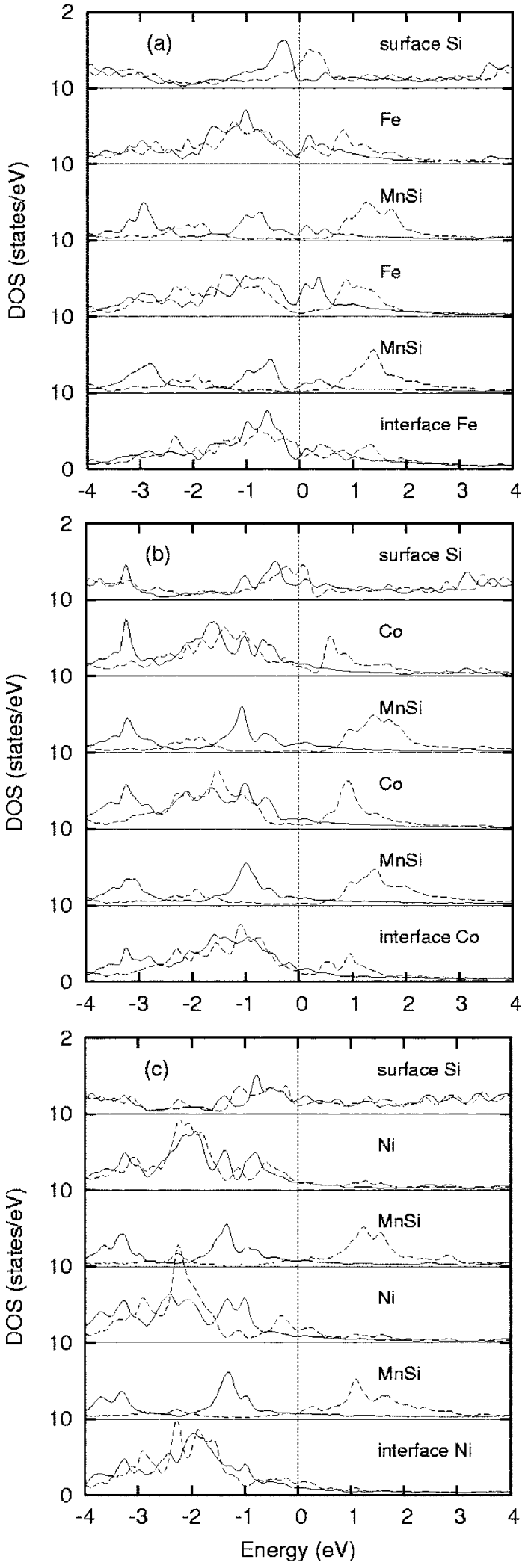

FIG. 6. The layer-resolved DOS of the Si-terminated threelayered $\mathrm{Fe}_{2} \mathrm{MnSi}$ (a), $\mathrm{Co}_{2} \mathrm{MnSi}$ (b) and $\mathrm{Ni}_{2} \mathrm{MnSi}$ (c) films on $\mathrm{Si}(001)$ with $M / \mathrm{Si}(M=\mathrm{Fe}, \mathrm{Co}$, or Ni) interface. In each panel, the overlayers are shown from surface (top) to interface (bottom) for the atomic structure depicted in Fig. 5(b). Full lines show the majority spin, dashed lines the minority spin component. of $219 \mathrm{~K}$ for $\mathrm{Fe}_{2} \mathrm{MnSi}, 985 \mathrm{~K}$ for $\mathrm{Co}_{2} \mathrm{MnSi}$, and 320, 344, and $380 \mathrm{~K}$ for $\mathrm{Ni}_{2} \mathrm{MnZ}$ ( $Z=\mathrm{Sn}, \mathrm{Ge}, \mathrm{Ga}$, respectively). ${ }^{2,3}$

Secondly, we analyze the spin magnetic moments in the films (see Table V). On the one hand, the Mn spin moment, being generally larger than $2 \mu_{B}$, increases in the $M_{2} \mathrm{MnSi}$ films as $M$ varies from $\mathrm{Fe}$ through $\mathrm{Co}$ to $\mathrm{Ni}$, following the same trend as in the bulk materials. This finding can be at least partly ascribed to decreasing $d$ - $d$ hybridization among $\mathrm{Mn}$ and the neighboring transition metal atoms when going from $\mathrm{Fe}$ to $\mathrm{Ni}$, in accordance with the increasing energy separation between the Mn $3 d$ and $M 3 d$ orbitals (see Fig. 2). On the other hand, one can argue that the Mn spin moment in the $M_{2} \mathrm{MnSi} / \mathrm{Si}(001)$ films is still smaller than that in the $M_{2} \mathrm{MnSi}$ bulk. Again, this can be explained by stronger inplane $d$ - $d$ hybridization in the film compared to the bulk, which gives rise to more delocalized planar electronic states and a reduced magnetic moment. The reason for this anisotropy is that the lattice constant of bulk $\mathrm{Si}$ is about $4 \%$ smaller than that of cubic $M_{2} \mathrm{MnSi}$. Hence the $M_{2} \mathrm{MnSi}$ films have reduced planar lattice constant under the epitaxial constraint. The transition metal atom $M(=\mathrm{Fe}, \mathrm{Co}, \mathrm{Ni})$ has a spin moment less than $1 \mu_{B}$. In addition, the $\mathrm{Si}$ atom in the $\mathrm{MnSi}$ layer has a small induced spin moment which is opposite to the spin moment of the neighboring metal atom, and generally smaller than $0.05 \mu_{B} / \mathrm{Si}$. The substrate $\mathrm{Si}$ atoms have an even smaller spin moment of less than $0.02 \mu_{B} / \mathrm{Si}$ oscillating in its orientation between one substrate layer and the next one.

For the Si-terminated three-layered $\mathrm{M}_{2} \mathrm{MnSi}$ films, our calculations find, in complete analogy to the above two-layer case, a FM metallic ground state irrespective of the nature of the transition metal. In addition to the strong FM Mn-Mn intralayer coupling discussed above, the interlayer Mn-Mn coupling (evaluated by switching the relative orientation of the magnetic moment in two neighboring MnSi layers in the supercell) is $4 \mathrm{meV} / \mathrm{Mn}$ in the $\mathrm{Fe}_{2} \mathrm{MnSi}$ film, $167 \mathrm{meV} / \mathrm{Mn}$ in $\mathrm{Co}_{2} \mathrm{MnSi}$, and $30 \mathrm{meV} / \mathrm{Mn}$ in $\mathrm{Ni}_{2} \mathrm{MnSi}$. The reduced interlayer coupling can be at least partly ascribed to a tetrago-

TABLE VI. Formation energies [Eq. (1)] and heat of reaction $\Delta E$ [Eq. (2)] [in unit of eV per $(1 \times 1)$ cell] of the Si-terminated two-layered $(2 L)$ and $3 L M_{2} \mathrm{MnSi} / \mathrm{Si}(001)$ films and of the MnSiterminated $1 L, 2 L$, and $3 L M_{2} \mathrm{MnSi} / \mathrm{Si}(001)$ films (see Fig. 5). All films have a $M / \mathrm{Si}$ interface.

\begin{tabular}{ccccccc}
\hline \hline & & \multicolumn{2}{c}{ Si-term. } & & \multicolumn{2}{c}{ MnSi-term. } \\
\cline { 3 - 4 } \cline { 6 - 7 } \cline { 5 - 6 } $1 L$ & $M$ & $E_{\text {form }}$ & $\Delta E$ & & $E_{\text {form }}$ & $\Delta E$ \\
\hline \multirow{2}{*}{$2 L$} & $\mathrm{Fe}$ & & & -0.20 & 0.86 \\
& $\mathrm{Co}$ & & & -0.71 & 0.92 \\
& $\mathrm{Ni}$ & & & -0.80 & 0.81 \\
& $\mathrm{Fe}$ & -1.08 & 0.02 & & -1.42 & 0.33 \\
& $\mathrm{Co}$ & -1.87 & 0.14 & & -2.30 & 0.53 \\
$3 L$ & $\mathrm{Ni}$ & -2.07 & 0.40 & & -2.37 & 0.62 \\
& $\mathrm{Fe}$ & -2.51 & -0.65 & & -2.87 & -0.66 \\
& $\mathrm{Co}$ & -3.48 & -0.05 & & -3.99 & 0.09 \\
& $\mathrm{Ni}$ & -3.42 & 0.58 & -3.69 & 0.48 \\
\hline \hline
\end{tabular}


nal distortion, by noting that the Heusler alloy film is under compressive epitaxial strain on $\mathrm{Si}(001)$, as stated above, and thus has an enlarged spacing between layers. In addition, the $M$ spin, which mediates the effective Mn-Mn coupling, plays an important role for the magnetic ordering. Note that in the three-layered $M_{2} \mathrm{MnSi}$ films, the $M$ atoms in the layer sandwiched between two MnSi layers have an averaged spin moment of $0.21 \mu_{B} / \mathrm{Fe}, 0.95 \mu_{B} / \mathrm{Co}$, and $0.28 \mu_{B} / \mathrm{Ni}$, as seen from Table V. In contrast to this, we observe that for the layered AFM ordering of the Mn spins, the Co spin in the middle layer is quenched to a value close to zero. The vanishing of the Co spin moment in the layered AFM state, sitting between two spin-antiparallel MnSi layers, is simply a consequence of symmetry. The highest energy cost of switching from FM to AFM alignment of the Mn spins correlates with the largest magnetic moment at Co in the FM state in the three Heusler alloys studied here. This indicates that the quenching of the Co spin moment is energetically unfavorable and hence the FM state is preferred over the AFM state.

Next, we investigate if the half-metallic properties of the $\mathrm{Co}_{2} \mathrm{MnSi}$ and $\mathrm{Fe}_{2} \mathrm{MnSi}$ bulk materials also show up in the thin films. In Fig. 6, the overlayer-resolved DOS of the Siterminated three-layered $M_{2} \mathrm{MnSi}(M=\mathrm{Fe}, \mathrm{Co}, \mathrm{Ni})$ films on $\mathrm{Si}(001)$ is shown. Generally, the films do not show a gap in the DOS at the Fermi level. However, the spin polarization at the Fermi level is high in the three middle layers, $\mathrm{MnSi}-\mathrm{Fe}-$ $\mathrm{MnSi}$ or $\mathrm{MnSi}-\mathrm{Co}-\mathrm{MnSi}$. We interpret this as an incipient recovery of the half-metallicity of the bulk $\mathrm{Fe}_{2} \mathrm{MnSi}$ and $\mathrm{Co}_{2} \mathrm{MnSi}$. However, in the $\mathrm{Ni}_{2} \mathrm{MnSi}$ film, this is not the case, consistent with our finding that bulk $\mathrm{Ni}_{2} \mathrm{MnSi}$ is not halfmetallic. In all the $\mathrm{M}_{2} \mathrm{MnSi}$ films studied here, the surface $\mathrm{Si}$ layer has a sizable spin polarization $(>30 \%)$ at the Fermi level, following the definition in Ref. 5, while the subsurface $M$ and the interfacial $M$ layers have only low spin polarization $(<10 \%)$ at the Fermi level (except for $\sim 20 \%$ for the interfacial $\mathrm{Ni}$ layer).

Finally, we turn to the subject of thermodynamic stability. By calculating the formation energy using Eq. (1), we conclude that all Si-terminated two- and three-layered $\mathrm{M}_{2} \mathrm{MnSi}$ films on $\mathrm{Si}(001)$ are stable against a decomposition into the clean $\mathrm{Si}(001)$ surface and bulk TMs. This is indicated by their negative $E_{\text {form }}$ values, as seen in Table VI. Moreover, we checked the stability of the $M_{2} \mathrm{MnSi}$ films against separated $M \mathrm{Si}$ and $\mathrm{MnSi}$ films by calculating the heat of reaction, $\Delta E$, defined by

$$
\begin{aligned}
& M \mathrm{Si} / \mathrm{Si}(001)+\mathrm{MnSi} / \mathrm{Si}(001) \\
& \quad \rightarrow M_{2} \mathrm{MnSi} / \mathrm{Si}(001)+\text { clean } \mathrm{Si}(001)+\Delta E .
\end{aligned}
$$

The $M_{2} \mathrm{MnSi}$ films are stable (unstable) if $\Delta E$ is positive (negative). As shown by our results summarized in Table VI, the two-layered $\mathrm{Fe}_{2} \mathrm{MnSi}$ film $[\Delta E=0.02 \mathrm{eV}$ per $(1 \times 1)$ cell $]$ is close to becoming unstable, and the three-layered one $[\Delta E=-0.65 \mathrm{eV}$ per $(1 \times 1)$ cell $]$ is obviously unstable. The two-layered $\mathrm{Co}_{2} \mathrm{MnSi}$ film is stable while the three-layered one tends to be unstable. However, the $\mathrm{Ni}_{2} \mathrm{MnSi}$ film is stable against a phase separation into the $\mathrm{NiSi}$ and $\mathrm{MnSi}$ films. This is because the NiSi film is less stable due to its
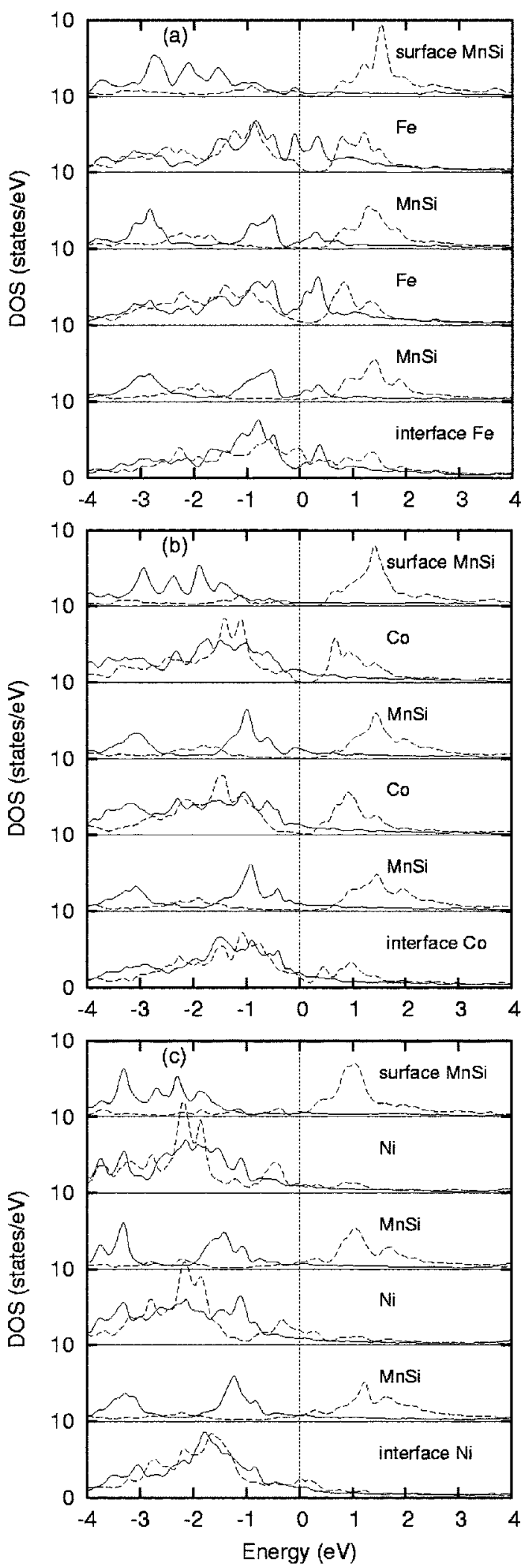

FIG. 7. The layer-resolved DOS of the MnSi-terminated threelayered $\mathrm{Fe}_{2} \mathrm{MnSi}$ (a), $\mathrm{Co}_{2} \mathrm{MnSi}$ (b) and $\mathrm{Ni}_{2} \mathrm{MnSi}$ (c) films on $\mathrm{Si}(001)$ with $M / \mathrm{Si}(M=\mathrm{Fe}, \mathrm{Co}$, or $\mathrm{Ni})$ interface. In each panel, the overlayers are shown from surface (top) to interface (bottom) for the atomic structure depicted in Fig. 5(e). Full lines show the majority spin, dashed lines the minority spin component. 
TABLE VII. Formation energies $[\mathrm{eV}$ per $(1 \times 1)$ cell] either of the Si- or MnSi-terminated $\mathrm{Co}_{2} \mathrm{MnSi} / \mathrm{Si}(001)$ films with a $\mathrm{MnSi} / \mathrm{Si}$ interface (see Fig. 5 but note that extra $\mathrm{Mn}$ atoms occupy the interstitial sites of the interfacial Si layer). The film thickness $(1 L, 2 L$, and $3 L)$ refers to the number of the Co-MnSi bilayers. The third column shows the heat of reaction $\Delta E[\mathrm{eV}$ per $(1 \times 1)$ cell], as defined in the text, Eq. (2). From the fourth column onwards, the overlayer-resolved (counted from the interface to the surface) atomic spin moments (in units of $\mu_{B}$ ) are shown. The substrate Si layers, each with an induced spin moment being generally less than $0.04 \mu_{B} / \mathrm{Si}$, are omitted.

\begin{tabular}{lccccccccc}
\hline \hline Si-term. & $E_{\text {form }}$ & $\Delta E$ & $\mathrm{MnSi}$ & $\mathrm{Co}$ & $\mathrm{MnSi}$ & $\mathrm{Co}$ & $\mathrm{MnSi}$ & $\mathrm{Co}$ & $\mathrm{Si}$ \\
\hline $1 L$ & -0.36 & 0.57 & & & & & $2.68 /-0.02$ & 0.78 & 0.01 \\
$2 L$ & -1.98 & 0.21 & & & $2.87 /-0.01$ & 0.98 & $2.79 /-0.04$ & 0.74 & 0.02 \\
$3 L$ & -3.54 & -0.36 & $2.77 /-0.01$ & 1.04 & $2.82 /-0.04$ & 1.02 & $2.83 /-0.04$ & 0.81 & 0.03 \\
\hline MnSi-term. & $E_{\text {form }}$ & $\Delta E$ & $\mathrm{MnSi}$ & $\mathrm{Co}$ & $\mathrm{MnSi}$ & $\mathrm{Co}$ & $\mathrm{MnSi}$ & $\mathrm{Co}$ & $\mathrm{MnSi}$ \\
$1 L$ & -0.92 & 1.09 & & & & & $2.74 /-0.02$ & 0.88 & $3.54 /-0.10$ \\
$2 L$ & -2.48 & 0.35 & & & $2.80 /-0.01$ & 1.02 & $2.78 /-0.05$ & 0.87 & $3.53 /-0.11$ \\
$3 L$ & -4.09 & -0.49 & $2.78 /-0.01$ & 1.03 & $2.82 /-0.04$ & 1.06 & $2.78 /-0.04$ & 0.90 & $3.53 /-0.11$ \\
\hline \hline
\end{tabular}

oversaturated eightfold $\mathrm{Si}$ coordination of $\mathrm{Ni}$, while the $\mathrm{Ni}_{2} \mathrm{MnSi}$ film is stable, involving only fourfold $\mathrm{Si}$ coordination of $\mathrm{Ni}$.

\section{2. $M_{2} \mathrm{MnSi} / \mathrm{Si}(001)$ : MnSi termination and $\mathrm{M} / \mathrm{Si}$ interface}

Next we deal with the $M_{2} \mathrm{MnSi} / \mathrm{Si}(001)$ thin films with MnSi termination [see Figs. 5(c)-5(e)]. The surface Mn atom has an increased spin moment of about $3.5 \mu_{B}$, and the surface $\mathrm{Si}$ atom also has an increased induced spin moment of about $-0.1 \mu_{B}$, as seen in Table V. The spin moments of $\mathrm{Mn}$ and $\mathrm{Si}$ in the sandwich layer between two $M$ layers are, due to the identical environment, very similar to those in the Si-terminated $M_{2} \mathrm{MnSi}$ films discussed above. The spin moment of the $M$ atom sandwiching two MnSi layers, which plays an important role in the effective Mn-Mn coupling, is less than $0.2 \mu_{B} / \mathrm{Fe}$, about $0.8-1.0 \mu_{B} / \mathrm{Co}$ or $0.2-0.3 \mu_{B} / \mathrm{Ni}$. These values agree closely with those of the Si-terminated three-layered $M_{2} \mathrm{MnSi}$ films discussed above, and of the bulk materials. The MnSi termination brings about a gain in the formation energy in the range of $0.3-0.5 \mathrm{eV}$ per $(1 \times 1)$ cell for the two- and three-layered $M_{2} \mathrm{MnSi}$ films (the exact value being materials-dependent) compared with the Si-terminated $M_{2} \mathrm{MnSi}$ films, which means that the former has higher thermodynamic stability. However, we would like to draw the reader's attention to the fact that the cohesive energy of $\mathrm{Si}$ is larger than that of $\mathrm{Mn}$ by about $1.5 \mathrm{eV}$, as indicated by experiments and our calculations. Combining the calculated values for the stability of both the films and the bulk phases, we conclude that the MnSi termination has highest thermodynamic stability mostly due to the low cohesive energy of Mn bulk. However, Si has a higher surface adsorption energy in the $\mathrm{Si}$ termination than $\mathrm{Mn}$ in the $\mathrm{MnSi}$ termination by about $1.0 \mathrm{eV}$. In this sense, the Si-terminated $M_{2} \mathrm{MnSi}$ films have stronger surface $\mathrm{Si}-M$ bonds than the $\mathrm{Mn}-M$ bonds present in the MnSi termination, and therefore the $\mathrm{Si}$ termination is chemically more stable. Moreover, as seen in Table VI, all the MnSi-terminated $M_{2} \mathrm{MnSi}$ films are stable against a phase separation, except for the three-layered $\mathrm{Fe}_{2} \mathrm{MnSi}$ film.
In Fig. 7, the overlayer-resolved DOS of the MnSiterminated three-layered $M_{2} \mathrm{MnSi}$ films are shown. The surface $\mathrm{MnSi}$ layer of the $\mathrm{Fe}_{2} \mathrm{MnSi}$ film brings about a notable change for the subsurface Fe layer compared to the Si termination, as seen in Fig. 6(a), and this Fe layer now becomes highly spin-polarized $(\sim 65 \%)$ at the Fermi level. The three middle layers $\mathrm{MnSi}-\mathrm{Fe}-\mathrm{MnSi}$, are less affected. Again, we observe a tendency to recover the bulk half-metallicity. In addition, the interfacial Fe layer has a considerable spin polarization $(\sim 45 \%)$ at the Fermi level. Similar changes occur in the MnSi-terminated $\mathrm{Co}_{2} \mathrm{MnSi}$ films. In particular, the surface $\mathrm{MnSi}$ layer and the other overlayers, except for the interfacial layer, become almost half-metallic. However, for the $\mathrm{Ni}_{2} \mathrm{MnSi}$ films, the surface $\mathrm{MnSi}$ layer brings no pronounced changes as compared with the Si termination.

\section{3. $\mathrm{Co}_{2} \mathrm{MnSi} / \mathrm{Si}(001): \mathrm{MnSi} / \mathrm{Si}$ interface}

When Mn atoms occupy the interstitial sites of the interfacial Si layer, as seen in Fig. 5, this layer now becomes a $\mathrm{MnSi} / \mathrm{Si}$ interface, replacing the former $\mathrm{Co} / \mathrm{Si}$ interface. Here we investigate $\mathrm{Co}_{2} \mathrm{MnSi} / \mathrm{Si}(001)$ films with this interface, considering two different surface terminations, either pure Si or MnSi termination. As seen in Table VII, the interfacial MnSi layer enhances the spin moments of the overlayers, especially of the near-interface Co layer, as compared with the $\mathrm{Co}_{2} \mathrm{MnSi} / \mathrm{Si}(001)$ film with the $\mathrm{Co} / \mathrm{Si}$ interface (Table V). Comparing films with the same number of Co atoms, we find that the $\mathrm{MnSi}$ interface makes the films slightly more stable, through lowering the formation energy by $0.2 \mathrm{eV}$ per $(1 \times 1)$ cell or less for $1 L, 2 L$, or $3 L$ thickness (see Tables VI and VII for comparison), as a result of the low cohesive energy of bulk Mn which favors incorporation of extra $\mathrm{Mn}$ atoms. However, the $\mathrm{Co} / \mathrm{Si}$ and $\mathrm{MnSi} / \mathrm{Si}$ interfaces of the $\mathrm{Co}_{2} \mathrm{MnSi} / \mathrm{Si}(001)$ film differ by less than $0.2 \mathrm{eV}$, implying that chemical disorder in the interface layer could occur easily through thermal fluctuations. In addition, $\mathrm{MnSi}$ termination goes along with a gain in formation energy, compared with Si termination, about $0.5 \mathrm{eV}$ per $(1 \times 1)$ cell for the one-, two-, and three-layered $\mathrm{Co}_{2} \mathrm{MnSi}$ films with 

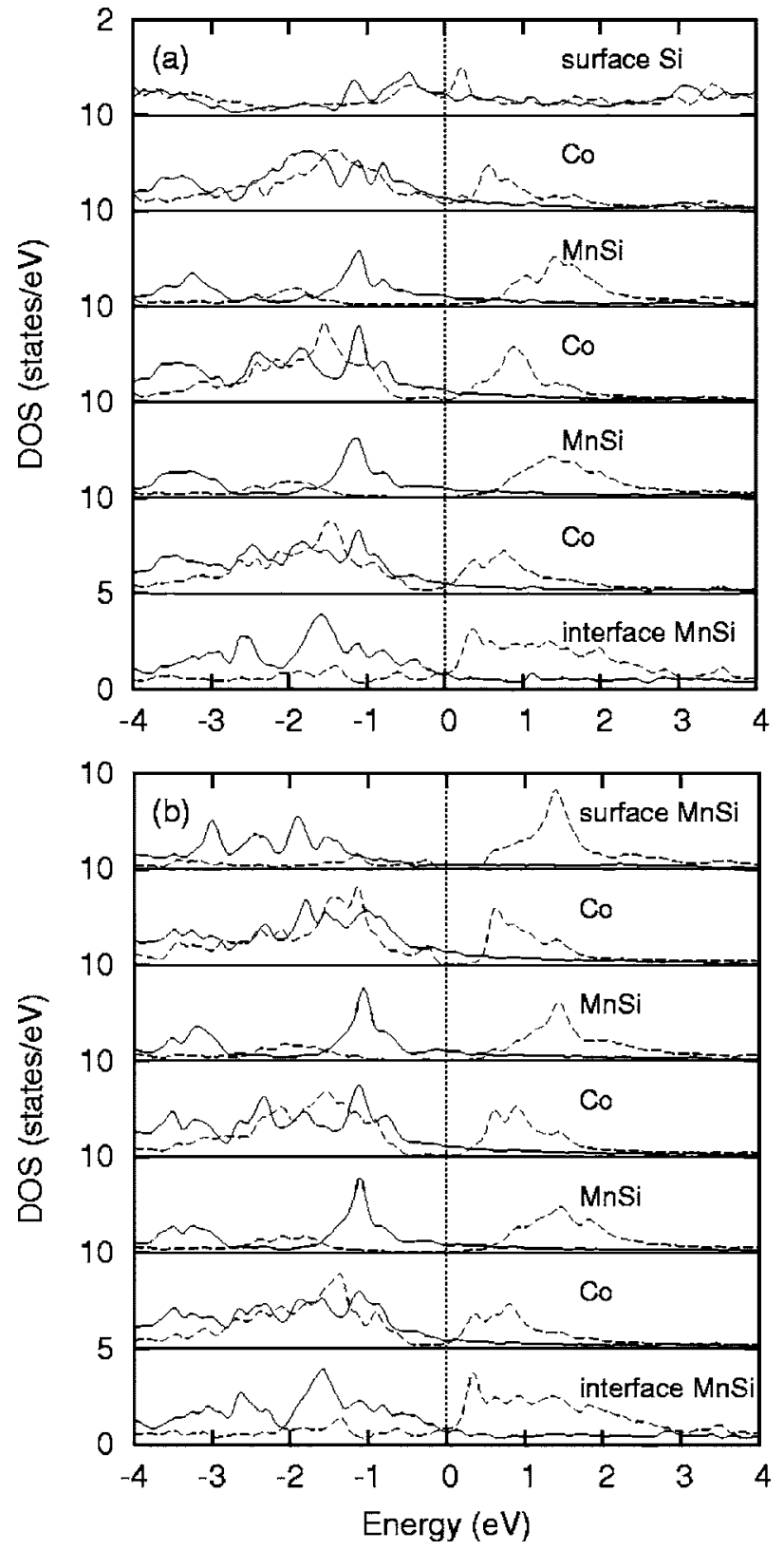

FIG. 8. The layer-resolved DOS of the Si-terminated (a) or MnSi-terminated (b) three-layered $\mathrm{Co}_{2} \mathrm{MnSi}$ films on $\mathrm{Si}(001)$ with $\mathrm{MnSi} / \mathrm{Si}$ interface. In each panel, the layers are shown from surface (top) to interface (bottom). Full lines show the majority spin, dashed lines the minority spin component.

$\mathrm{MnSi} / \mathrm{Si}$ interface, following the same trend as in the $\mathrm{Co}_{2} \mathrm{MnSi} / \mathrm{Si}(001)$ film with the $\mathrm{Co} / \mathrm{Si}$ interface. In Fig. 8, the overlayer-resolved DOS of both the $\mathrm{Si}$ - and the $\mathrm{MnSi}$ terminated three-layered $\mathrm{Co}_{2} \mathrm{MnSi} / \mathrm{Si}(001)$ films with $\mathrm{MnSi} / \mathrm{Si}$ interface are shown. Although the interfacial Mn atom has almost the same spin moment as the middle $\mathrm{MnSi}$ layers where bulk half-metallicity is almost recovered, we observe that the spin polarization at the Fermi level in the interface layer is still tiny $(<10 \%)$. Hence, in this respect, the $\mathrm{MnSi} / \mathrm{Si}$ interface brings no pronounced change for the overlayers as compared to the $\mathrm{Co}_{2} \mathrm{MnSi} / \mathrm{Si}(001)$ film with $\mathrm{Co} / \mathrm{Si}$ interface.

\section{CONCLUSION}

In summary, we have presented systematic DFT-GGA calculations for pseudomorphic thin films of monosilicides $M \mathrm{Si}$ $(M=\mathrm{Mn}, \mathrm{Fe}, \mathrm{Co}, \mathrm{Ni})$ with $\mathrm{CsCl}$-like atomic structure, and for thin films of Heusler alloys $M_{2} \mathrm{MnSi}(M=\mathrm{Fe}, \mathrm{Co}, \mathrm{Ni})$ on $\mathrm{Si}(001)$, with particular focus on the trends within the transition metal series.

Our calculations show that for pseudomorphic $M$ Si films on $\mathrm{Si}(001)$, Si surface termination is energetically preferred because it optimizes the surface valence bond structure, i.e., fourfold coordination of surface $\mathrm{Si}$ and seven- or eight-fold coordination of subsurface $M$ atoms are achieved. The $M-\mathrm{Si}$ chemical bond becomes stronger as $M$ varies from $\mathrm{Mn}$ through $\mathrm{Fe}$ and $\mathrm{Co}$ to $\mathrm{Ni}$, due to decreasing $M 3 d-\mathrm{Si} 3 s 3 p$ energy separation, and hence increasing hybridization of the metal $3 d$ states with the $\mathrm{Si}$ valence band. The calculated variations in thermodynamic stability of the $M \mathrm{Si} / \mathrm{Si}(001)$ films can be accounted for in terms of both the $M 3 d-\mathrm{Si} 3 s 3 p$ energy separation and the $M 3 d$ orbital occupation.

These trends for the bond strength also enable us to rationalize the observed atomic ordering in Heusler alloys and to explain the experimentally observed site preference of transition metal impurities added to Heusler alloys. We confirm previous work ${ }^{11}$ showing that CoSi films, in addition to ultrathin FM MnSi films, ${ }^{5}$ are another possibility to grow thin FM silicide films on $\mathrm{Si}(001)$, while FeSi and NiSi films are found to be nonmagnetic. Therefore, MnSi and CoSi films on $\mathrm{Si}(001)$ deserve further experimental studies.

For the $\mathrm{M}_{2} \mathrm{MnSi} / \mathrm{Si}(001)$ films, our results show that MnSi termination is thermodynamically stable. The slightly less stable Si termination, once formed, is long lived, since removing $\mathrm{Si}$ atoms is energetically more costly than removing $\mathrm{Mn}$ atoms. Except for the atoms in the surface and interface layers, we find that the electronic structure known from the bulk samples is recovered quickly in the interior of the overlayers. In particular, the half-metallicity of bulk $\mathrm{Fe}_{2} \mathrm{MnSi}$ and $\mathrm{Co}_{2} \mathrm{MnSi}$ is almost recovered in the three middle layers of the films investigated. As far as magnetic ordering in the $\mathrm{M}_{2} \mathrm{MnSi}$ films is concerned, we find that the effective intralayer Mn-Mn FM couplings mediated by the first-neighbor $M$ atoms are strong and approximately scale with the measured Curie temperatures of the corresponding bulk $M_{2} \mathrm{MnSi}$ samples. The interlayer Mn-Mn FM coupling remains strong in the $\mathrm{Co}_{2} \mathrm{MnSi}$ films while it is (much) reduced in the $\mathrm{Ni}_{2} \mathrm{MnSi}\left(\mathrm{Fe}_{2} \mathrm{MnSi}\right)$ films. The $\mathrm{Co}_{2} \mathrm{MnSi} / \mathrm{Si}(001)$ thin film is thermodynamically stable and has a robust FM metallic ground state, and thus is most relevant for possible applications. However, by analyzing our calculations we also identify two effects that could possibly be detrimental for use of these films for spin injection. The $\mathrm{Co} / \mathrm{Si}$ and $\mathrm{MnSi} / \mathrm{Si}$ interfaces are found to have a similar formation energy, which makes thermally induced interfacial disorder likely and the interfacial Co or MnSi layer does not display the gap in the layer-resolved DOS of the minority spin channel characteristic for a half-metal.

\section{ACKNOWLEDGMENT}

This work was supported by the Deutsche Forschungsgemeinschaft through SFB 290. 
${ }^{1}$ I. Žutić, J. Fabian, and S. D. Sarma, Rev. Mod. Phys. 76, 323 (2004).

${ }^{2}$ J. Kübler, A. R. Williams, and C. B. Sommers, Phys. Rev. B 28, 1745 (1983).

${ }^{3}$ S. Fujii, S. Ishida, and S. Asano, J. Phys. Soc. Jpn. 63, 1881 (1994).

${ }^{4}$ I. Galanakis, P. H. Dederichs, and N. Papanikolaou, Phys. Rev. B 66, 174429 (2002).

${ }^{5}$ H. Wu, M. Hortamani, P. Kratzer, and M. Scheffler, Phys. Rev. Lett. 92, 237202 (2004).

${ }^{6}$ N. Manyala, Y. Sidis, J. F. Ditusa, G. Aeppli, D. P. Young, and Z. Fisk, Nat. Mater. 3, 255 (2004).

${ }^{7}$ M. K. Chattopadhyay, S. B. Roy, and S. Chaudhary, Phys. Rev. B 65, 132409 (2002).

${ }^{8}$ E. G. Moroni, R. Podloucky, and J. Hafner, Phys. Rev. Lett. 81, 1969 (1998).

${ }^{9}$ H. von Känel, K. A. Mäder, E. Müller, N. Onda, and H. Sirringhaus, Phys. Rev. B 45, 13807 (1992).

${ }^{10}$ H. von Känel, C. Schwarz, S. Goncalves-Conto, E. Müller, L. Miglio, F. Tavazza, and G. Malegori, Phys. Rev. Lett. 74, 1163 (1995).

${ }^{11}$ G. Profeta, S. Picozzi, A. Continenza, and R. Podloucky, Phys. Rev. B 70, 235338 (2004); G. Profeta, S. Picozzi, A. Continenza, G. Schneider, and R. Podloucky, J. Magn. Magn. Mater. 272-276, e233 (2004).

${ }^{12}$ G. M. Dalpian, A. J. R. da Silva, and A. Fazzio, Surf. Sci. 566568, 688 (2004)

${ }^{13}$ A. P. Horsfield, S. D. Kenny, and H. Fujitani, Phys. Rev. B 64, 245332 (2001).

${ }^{14}$ S. Higai and T. Ohno, Phys. Rev. B 62, R7711 (2000).

${ }^{15}$ S. Kämmerer, S. Heitmann, D. Meyners, D. Sudfeld, A. Thomas, A. Hütten, and G. Reiss, J. Appl. Phys. 93, 7945 (2003).

${ }^{16}$ S. Kämmerer, A. Thomas, A. Hütten, and G. Reiss, Appl. Phys. Lett. 85, 79 (2004).

${ }^{17}$ J. Schmalhorst, S. Kämmerer, M. Sacher, G. Reiss, A. Hütten, and A. Scholl, Phys. Rev. B 70, 024426 (2004).

${ }^{18}$ T. Ambrose, J. J. Krebs, and G. A. Prinz, J. Appl. Phys. 87, 5463 (2000).

${ }^{19}$ W. H. Wang, M. Przybylski, W. Kuch, L. I. Chelaru, J. Wang, Y. F. Lu, J. Barthel, H. Meyerheim, and J. Kirschner, Phys. Rev. B 71, 144416 (2005).

${ }^{20}$ I. Galanakis, J. Phys.: Condens. Matter 14, 6329 (2002).

${ }^{21}$ S. J. Hashemifar, P. Kratzer, and M. Scheffler, Phys. Rev. Lett. 94, 096402 (2005).

${ }^{22}$ S. Picozzi, A. Continenza, and A. J. Freeman, J. Phys. Chem. Solids 64, 1697 (2003).

${ }^{23}$ S. Picozzi, A. Continenza, and A. J. Freeman, J. Appl. Phys. 94, 4723 (2003).

${ }^{24}$ P. Blaha, K. Schwarz, G. K. H. Madsen, D. Kvasnicka, and J. Luitz, WIEN2K, an Augmented Plane Wave plus Local Orbitals Program for Calculating Crystal Properties, K. Schwarz, Techn.
Univ. Wien, Austria, 2001

${ }^{25}$ J. P. Perdew, K. Burke, and M. Ernzerhof, Phys. Rev. Lett. 77, 3865 (1996).

${ }^{26}$ P. H. T. Philipsen and E. J. Baerends, Phys. Rev. B 54, 5326 (1996).

${ }^{27}$ E. G. Moroni, W. Wolf, J. Hafner, and R. Podloucky, Phys. Rev. B 59, 12860 (1999).

${ }^{28}$ Using of the same $R K_{\max }=7.8$ as in our previous work (Ref. 5) but a little smaller $R_{\mathrm{Si}}=R_{\mathrm{Fe}}=R_{\mathrm{Co}}=R_{\mathrm{Ni}}=1.06 \AA$ (2.0 bohr) yields the higher cut-off energy of 15.2 Ryd, compared with that of 13.8 Ryd used previously. Consistency between our presently calculated results of the $\mathrm{Mn} / \mathrm{Si}(001)$ system and those previously obtained, as seen in Table I, indicates that the technical settings used here ensure reliable numerical accuracy of our results.

${ }^{29}$ I. I. Mazin, Phys. Rev. Lett. 83, 1427 (1999).

${ }^{30}$ R. Panguluri, G. Tsoi, B. Nadgorny, S. H. Chun, N. Samarth, and I. I. Mazin, Phys. Rev. B 68, 201307(R) (2003).

${ }^{31}$ D. van der Marel, A. Damascelli, K. Schulte, and A. A. Menovsky, Physica B 224, 138 (1998).

${ }^{32}$ S. Walter, R. Bandorf, W. Weiss, K. Heinz, U. Starke, M. Strass, M. Bockstedte, and O. Pankratov, Phys. Rev. B 67, 085413 (2003).

${ }^{33}$ P. Bertoncini, P. Wetzel, D. Berling, G. Gewinner, C. UlhaqBouillet, and V. P. Bohnes, Phys. Rev. B 60, 11123 (1999).

${ }^{34}$ T. J. Burch, J. I. Budnick, V. A. Niculescu, K. Raj, and T. Litrenta, Phys. Rev. B 24, 3866 (1981).

35 The FM ground state both of the CsCl-like CoSi bulk and the thin film on $\mathrm{Si}(001)$ is closely correlated with the crystal structure (eightfold coordination) and the peculiar electronic band structure. Note that the natural CoSi at the B20 phase (sevenfold coordination) is found to be NM both in experiments and in our calculation. Moreover, our calculations show that for the CsCllike $\mathrm{CoSi}$ thin film on $\mathrm{Si}(111)$, the 2(Si-Co)/Si(111) with sevenfold coordination is $\mathrm{NM}$, while the $3(\mathrm{Si}-\mathrm{Co}) / \mathrm{Si}(111)$ with eightfold coordination is FM.

${ }^{36}$ M. P. Raphael, B. Ravel, M. A. Willard, S. F. Cheng, B. N. Das, R. M. Stroud, K. M. Bussmann, J. H. Claassen, and V. G. Harris, Appl. Phys. Lett. 79, 4396 (2001).

${ }^{37}$ L. Ritchie, G. Xiao, Y. Ji, T. Y. Chen, C. L. Chien, M. Zhang, J. Chen, Z. Liu, G. Wu, and X. X. Zhang, Phys. Rev. B 68, 104430 (2003).

${ }^{38}$ L. J. Singh, Z. H. Barber, Y. Miyoshi, Y. Bugoslavski, W. R. Branford, and L. F. Cohen, Appl. Phys. Lett. 84, 2367 (2004).

${ }^{39}$ S. Ishida, S. Fujii, S. Kashiwagi, and S. Asano, J. Phys. Soc. Jpn. 64, 2152 (1995).

${ }^{40}$ S. Picozzi, A. Continenza, and A. J. Freeman, Phys. Rev. B 66, 094421 (2002).

${ }^{41}$ S. Fujii, S. Ishida, and S. Asano, J. Phys. Soc. Jpn. 64, 185 (1995).

${ }^{42}$ P. Mohn and E. Supanetz, Philos. Mag. B 78, 629 (1998). 